\title{
Finite-Time Observer-Based Adaptive Control of Switched System with Unknown Backlash-Like Hysteresis
}

\author{
Guofa Sun (iD) and Yaming Xu (iD \\ School of Information and Control Engineering, Qingdao University of Technology, Qingdao 266520, China \\ Correspondence should be addressed to Yaming Xu; xuyaming2000@163.com
}

Received 24 July 2019; Revised 6 September 2019; Accepted 26 September 2019; Published 29 October 2019

Guest Editor: Chun Wei

Copyright ( 2019 Guofa Sun and Yaming Xu. This is an open access article distributed under the Creative Commons Attribution License, which permits unrestricted use, distribution, and reproduction in any medium, provided the original work is properly cited.

\begin{abstract}
This work investigates a finite-time observer problem for a class of uncertain switched nonlinear systems in strict-feedback form, preceded by unknown hysteresis. By using a finite-time performance function, a finite-time switched state observer (FTSO) is derived using radial basis function neural networks (RBFNNs) to estimate the unmeasured states. An adaptive feedback neural network tracking control is derived based on the backstepping technique, which guarantees that all the signals of the closed-loop system are bounded, the output tracking error converges to zero, and the observer error converges to a prescribed arbitrarily small region within a finite-time interval. In addition, two simulation studies and an experiment test are provided to verify the feasibility and effectiveness of the theoretical finding in this study.
\end{abstract}

\section{Introduction}

In the past decades, great attentions and developments have been gained in finite-time adaptive control design, which developed a great number of typical design approaches in the literature (see, e.g., [1-15]). A variety of different types of complex nonlinear systems have been explored by using neural networks or the fuzzy logic system-based adaptive backstepping technique. For instance, in [1-3], by utilizing the finite-time stability theory, barrier Lyapunov functions, and the adaptive backstepping method, various finite-time adaptive control strategies were proposed with unknown dead-zone, unknown hysteresis, and unknown time-delayed in the nonlinear system, respectively. Considering the full-state constraints in the nonlinear systems, an adaptive fuzzy controller was constructed to address the finite-time tracking control problem for a class of strict-feedback nonlinear systems in [4]. Besides, Wang et al. [5] developed a novel adaptive neural finite-time control strategy which considered quantized problems for single-input and single-output quantized nonlinear systems. Meanwhile, growth condition assumption was removed under the presented control approach. That assumption was also eliminated in [6]. Li et al.
[6] presented a finite-time fuzzy adaptive output feedback control for the first time for nonstrict-feedback nonlinear systems, while it overcame the problem of explosion of complexity by using the dynamic surface control technique. Moreover, Yu et al. [7] investigated an adaptive finite-time quantized tracking problem with input and output quantization and asymmetric actuator saturation, and they designed an observer-based adaptive finite-time quantized tracking controller at the same time. In [8], Liu et al. studied a finite-time adaptive fuzzy tracking control problem and proposed a finite-time adaptive state feedback fuzzy tracking controller with a new concept named finite-time performance function. Although a novel assessment standard of the finite-time semiglobal practical stability (SGPS) was found in [1-8], the main innovations of those literatures are designed controllers for nonswitched systems. Sui et al. [9] solved a finite-time switching control issue for nonstrictfeedback nonlinear switched systems, and they also constructed a novel finite-time SGPS for switched systems. Meanwhile, by utilizing a comparison theorem and a mean value theorem of integrals, a significant finite-time stability criterion for stochastic nonlinear systems was first set up in [10]. Chen et al. [11] studied an adaptive finite-time 
synchronization controller given for multiple robotic manipulators. It was worth noting that there existed some controllers to solve finite-time control problems in [12-15].

As a class of nonlinear inputs, hysteresis widely exists in the industrial system. Besides, the nonlinear characteristics of backlash-like hysteresis could seriously affect tracking performance, and it may cause a severe effect on the whole system. To capture hysteresis dynamics and achieve precise control, many scholars have made efforts to eliminate its effect. The hysteresis models can be roughly divided into two classes in [16, 17]: the operator-based hysteresis model [17-19] and the differential equation-based hysteresis model, such as Bouc-Wen model [20, 21] and backlash-like model [22-26]. For instance, in [18], Mayergoyz described a new approach to the scalar Preisach model of hysteresis which emphasized its phenomenological nature and mathematical generality. Zhang et al. [19] designed a hysteresis compensator to compensate the hysteresis nonlinearity described by a generalized PI hysteresis model, and the Krasnoselskii-Pokrovskii model was applied in [17]. The modeling accuracy can be guaranteed by increasing the number of superposed elementary operators for the operator-based hysteresis model, but also cause the computational burden during implementation. We continue to investigate the backlash-like hysteresis model in this paper since it has fewer parameters and has analytical solution. In [22], Ma et al. dealt with adaptive control for a class of switched nonlinear systems preceded by unknown backlashlike hysteresis, where the hysteresis was modeled by a differential equation. Similarly, Li et al. [23] investigated an adaptive neural output feedback control for a class of nonlinear systems with unknown backlash-like hysteresis of the actuator. Zhang and Lin [24] proposed a robust adaptive DSC control for a class of uncertain perturbed strict-feedback nonlinear systems preceded by unknown backlash-like hysteresis. On one hand, it is an active issue to study the systems with hysteresis nonlinearities which exist widely in the practical systems, such as manipulator system $[22,27]$, four-motor servomechanism [28, 29], and electrical circuit [30]. On the other hand, to date, there are no systematic methods to achieve a satisfactory result on finite-time switched state estimate of switched nonlinear systems with backlashlike hysteresis, which motivates our research interest. Hence, considering the inevitability of the backlash-like hysteresis constraint in many practical applications, it is worth further studying how to design a finite-time switched state observer (FTSO) for switched strict-feedback nonlinear systems with unknown backlash-like hysteresis.

During the development of control and applications, there exists a design algorithm which links observer design and hysteresis compensation. In [31], a novel hysteresis compensation method based on extended high-gain observer was presented without any specific hysteresis models. Besides, there exist several seminal observers designed with a system of hysteresis results [30, 32-34]. Huo et al. proposed an MIMO switched fuzzy observer to estimate the system states with hysteresis nonlinearities, which was less conservative than using a common observer for all subsystems. As stated in [33], they addressed the consensus tracking problem of a class of nonlinear multiagent system with hysteresis. And then, they developed a distributed adaptive neural output feedback control scheme proposed by constructing a state observer and using the backstepping technique. In order to improve the functional approximation capability and disturbance compensation ability for a system with unknown backlash-like hysteresis, Wang et al. [34] considered the coupler design between the radial basis function neural network and observer. Wei et al. [30] presented an extremum seeking algorithm to accurately estimate the state-of-power by an electrical circuit incorporating hysteresis effect. However, those contributions in [30-34] do not include finite-time convergence of observers, which further improves the tracking effect of the control strategy.

This study considers the finite-time adaptive switched observer problem for a kind of nonlinear systems with unknown hysteresis from a new perspective. Based on RBFNN approximation and finite-time performance function, the FTSO is proposed, which guarantees that observer error converge to a small neighborhood of the origin point in finite-time. Meanwhile, an adaptive tracking neural network controller is designed by the backstepping technique, which guarantees that all the signals of the switched closed-loop system are bounded and the observation error is converged to a small neighborhood of the origin point in a finite time. Compared with the existing results, the prominent contributions of this work are as follows:

(1) This paper proposes design process of a novel finitetime switched state observer which is designed to improve control performances for the switched system with unknown backlash-like hysteresis. In contrast with [1-15] which studies finite-time adaptive tracking controller, the smaller tracking error will be achieved with an FTSO for the same controller. In addition, with the presented control approach, all known state assumptions are removed by using the FTSO.

(2) To the best of our knowledge, it is the first time that the finite-time convergence problem of observer error is taken into consideration in a class of uncertain switched nonlinear systems with unknown backlash-like hysteresis, by using a finite-time performance function to obtain a better tracking performance. For switched systems with unknown backlash-like hysteresis, a control strategy with the FTSO can be applied more effectively and exactly.

This paper is organized as follows. Sections 2 presents the problem and the preliminaries. In Section 3, an adaptive finite-time switched state observer is designed. Section 4 draws control design and stability analysis. Simulation studies are conducted in Section 5 to illustrate the effectiveness of the proposed scheme. Finally, the paper is ended by concluding remarks in Section 6 .

\section{Problem Formulation and Preliminaries}

This section begins by presenting the control problem for a class of uncertain switched nonlinear systems with unknown 
backlash-like hysteresis. Then, it reviews some preliminaries about RBFNNs to facilitate the proposed observer and controller design procedure. Finally, a finite-time performance function is given to ensure observer error finite-time convergence.

2.1. Proposed System Descriptions. Consider a class of switched strict-feedback nonlinear systems with unknown hysteresis in the form of

$$
\left\{\begin{array}{l}
\dot{x}_{i}=g_{i, \sigma(t)} x_{i+1}+f_{i, \sigma(t)}\left(\bar{x}_{i}\right)+d_{i, \sigma(t)}(t), \\
\dot{x}_{n}=g_{n, \sigma(t)} u(v)+f_{n, \sigma(t)}\left(\bar{x}_{n}\right)+d_{n, \sigma(t)}(t), \\
y=x_{1},
\end{array}\right.
$$

where $\bar{x}_{i}=\left[x_{1}, x_{2}, \cdots, x_{i}\right]^{T} \in R^{i}, i=1,2, \cdots, n-1$, denotes the vectors of system states, $y \in R$ is the output of the switched nonlinear system, $f_{i}(\cdot)$ are smooth unknown nonlinear functions, and $g_{i}$ are known constants and $g_{i} \neq 0$. $\sigma(t):[0, \infty) \longrightarrow M=\{1, \ldots, m\}$ expressing the switching signal, which is a piecewise right continuous function. In addition, $\sigma(t)=j$ denotes the $j^{\text {th }}$ subsystem. $d_{i}(t)$ stands for the bounded external disturbance. $u(v) \in R$ is the output signal of backlash-like hysteresis described as

$$
\dot{u}=\alpha|\dot{v}|(c v-u)+b \dot{v},
$$

where $v$ indicates the input of the unknown backlashlike hysteresis. $\alpha, c$, and $b$ are unknown constants with $c>b$.

It follows from the analysis in $[22,23,35]$ that the solution of system (2) is expressed as

$$
u(t)=c v(t)+l(v)
$$

where $l(v)$ is bounded and satisfies

$$
l(v)=\left(u_{0}-c v_{0}\right) e^{-\alpha\left(v-v_{0}\right) \operatorname{sgn} \dot{v}}+e^{-\alpha v \operatorname{sgn} \dot{v}} \int_{v_{0}}^{v}(b-c) e^{\alpha \xi \operatorname{sgn} \dot{v}} d \xi,
$$

where $u_{0}$ and $v_{0}$ are the initial values of $u$ and $v$, respectively.

Figure 1 shows that a typical backlash-like hysteresis is generated. By (3), rewrite (1) as

$$
\left\{\begin{array}{l}
\dot{x}_{i}=g_{i, j} x_{i+1}+f_{i, j}\left(\bar{x}_{i}\right)+d_{i, j}(t), \\
\dot{x}_{n}=b_{0, j} v(t)+F_{n, j}\left(\bar{x}_{n}\right)+d_{n, j}(t), \\
y=x_{1},
\end{array}\right.
$$

with $F_{n}\left(\bar{x}_{n, j}\right)=f_{n, j}\left(\bar{x}_{n}\right)+g_{n, j} l(v)$ and $b_{0, j}=g_{n, j} c$.

This paper aims to derive an FTSO-based adaptive neural control signal $v$ for nonlinear system (1) with unknown backlash-like hysteresis (2), so that the following objectives can be achieved:

(1) Observer errors converge to an arbitrarily small specified neighborhood at a finite-time

(2) All the variables of the closed-loop system are bounded, and the tracking errors could converge to an arbitrarily small specified neighborhood

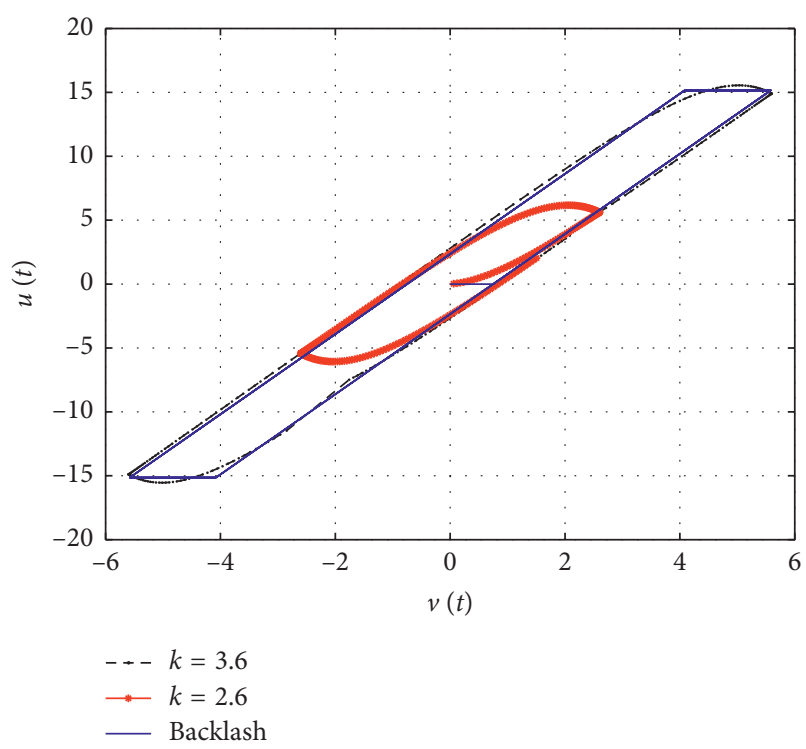

FIGURE 1: Hysteresis curves given by (2) with $\alpha=1, c=3.1635$, and $b=0.345$ for $v(t)=k \sin (2.3 t)$ with $k=2.6$ (red solid line) and $k=3.6$ (dashed line) to approximate backlash (blue solid line).

Lemma 1 (see $[36,37])$. For a nonlinear switching system of $t \in\left[0, T_{f}\right], N_{\sigma}\left(t, T_{f}\right)$ denotes the number of times and $\sigma(t)$ switches within a time interval $\left[0, T_{f}\right]$. If

$$
N_{\sigma}\left(t, T_{f}\right) \leq N_{0}+\frac{T_{f}-t}{\tau_{a}},
$$

where $\tau_{a}$ and $N_{0}$ are positive constants, then $\tau_{a}$ is average dwell time. In order to facilitate calculation, the buffeting bound $N_{0}$ is generally taken.

Lemma 2 (see $[36,37])$. A Lyapunov function candidate is defined as

$$
V_{P}=x^{T} P_{\sigma(t)} x, \quad \forall x \in R^{n},
$$

where $P_{\sigma(t)}$ is switched in accordance with the piecewise constant switching signal $\sigma(t)$. Then, the following properties are obtained:

(1) Each $V_{P \sigma(t)}=x^{T} P_{\sigma(t)} x$ in (7) is continuous. There exist constant scalars $\chi_{2} \geq \chi_{2} \geq 0$ such that

$\chi_{1}(\|x\|) \leq V_{P j}(x) \leq \chi_{2}(\|x\|), \quad \forall x \in R^{n}, \forall j \in M$.

(2) There exist constant scalars $\zeta \geq 1$ such that

$$
V_{P i}(x) \leq \zeta V_{P j}(x), \quad \forall x \in R^{n}, \forall i, j \in M .
$$

2.2. Radial Basis Function Neural Networks. The RBFNN has the ability to approximate the unknown smooth function $F(X): R^{q} \longrightarrow R$, and the form of $F(X)$ is as follows:

$$
F(X)=\theta^{T} \phi(X)+\eta(X)
$$


where $X=\left[X_{1}, \cdots, X_{q}\right]^{T} \in R^{q}$ and $\theta \in R^{q}$ are the weight vectors of the neural network, $\phi(X)=\left[\phi_{1}(X), \cdots, \phi_{q}(X)\right]^{T} \epsilon$ $R^{q}$ are the basis function vector of the neural network, and $\eta(X)$ is approximation error of neural network function, and $\eta(X)$ satisfies that $|\eta(X)|<\eta^{*}$, where $\eta^{*}$ is the upper bound of the estimated error.

In the following, $\phi$ is used to represent $\phi(X)$ and $\eta$ is used to represent $\eta(X)$, where the estimated error of the observer and the controller are expressed as $\eta_{o}$ and $\eta_{C}$, respectively.

The RBFNN is an approximator for nonlinear smooth function $f_{i}\left(\bar{x}_{i}\right)$ which is approximated as

$$
\begin{aligned}
\hat{f}_{i}\left(\hat{\bar{x}}_{i} \mid \hat{\theta}_{i}\right) & =\theta_{i}^{T} \phi_{i}\left(\hat{\bar{x}}_{i}\right), \\
\hat{\bar{x}}_{i} & =\left[y, x 2, \cdots, x_{n}\right]^{T},
\end{aligned}
$$

where $\overline{\bar{x}}_{i}$ is the estimation of $\bar{x}_{i}$.

The ideal parameter vectors $\theta_{i}^{*}$ are defined as

$$
\theta_{i}^{*}=\arg \min _{\theta_{i} \in \Omega_{i}}\left[\sup _{\substack{\hat{x} \in U_{i} \\ \mid}}\left|\hat{f}_{i}\left(\hat{\bar{x}}_{i} \mid \hat{\theta}_{i}\right)-f_{i}\left(\bar{x}_{i}\right)\right|\right],
$$

where $U_{i}$ and $\Omega_{i}$ are compact regions for $\hat{x}_{i}$ and $\theta_{i}$, respectively. Define the minimum approximation error $\eta_{i}$ as

$$
\eta_{i}=\hat{f}_{i}\left(\hat{\bar{x}}_{i} \mid \theta_{i}^{*}\right)-f_{i}\left(\bar{x}_{i}\right) .
$$

Lemma 3 (see [38]). The following inequality holds

$$
\phi^{T}(X) \phi(X) \leq \frac{1}{\phi^{T}\left(\bar{x}_{i}\right) \phi\left(\bar{x}_{i}\right)},
$$

for any inter $i \leq n$, where $\phi\left(\bar{x}_{i}\right)=\left[\pi_{1}\left(\bar{x}_{i}\right), \pi_{2}\left(\bar{x}_{i}\right), \cdots, \pi_{N}\left(\bar{x}_{i}\right)\right]^{T} /$ $\sum_{k=1}^{N} \pi_{k}\left(\bar{x}_{i}\right)$ and $\pi_{k}\left(\bar{x}_{i}\right)=\exp \left[-\left(\bar{x}_{i}-l_{i}\right)^{T}\left(\bar{x}_{i}-l_{i}\right) / 2 \gamma_{i}^{2}\right]$.

\subsection{Finite-Time Performance Function}

Definition 1. Different from a classical prescribed performance function in [39], a smooth function $\xi(t)$ is called finite-time performance function (FTPF) for all $\xi(0)=\xi_{0}$. There exists a settling time $0<T_{0}<\infty$, such that $\xi(t)<\xi\left(T_{0}\right)=\xi_{T_{0}}$, which is an arbitrarily small positive number. $\forall t \geq T_{0}=\xi(t)$, in this work, is defined by

$$
\xi(t)= \begin{cases}(\xi(0)-\tau \lambda t)^{1 / \tau}+\xi_{T_{0}}, & 0 \leq t \leq T_{0}, \\ \xi_{T_{0}}, & t>T_{0},\end{cases}
$$

where $\lambda, \tau, \xi(0)$, and $\xi_{T_{0}}$ are chosen as positive constants, and let $\tau=(q / p) \in(0,1]$, where $p$ and $q$ are chosen positive constants. Moreover, it has $T_{0}=\xi(0) / \tau \lambda$.

\section{Finite-Time Switched State Observer Design}

In this section, an FTSO is derived, involving the use of FTPF. Stability of the developed observer, estimating the states of switched nonlinear system (1) with unknown hysteresis (2), is proved by Lyapunov function.

For the switched nonlinear system (5), an FTSO is designed as follows:

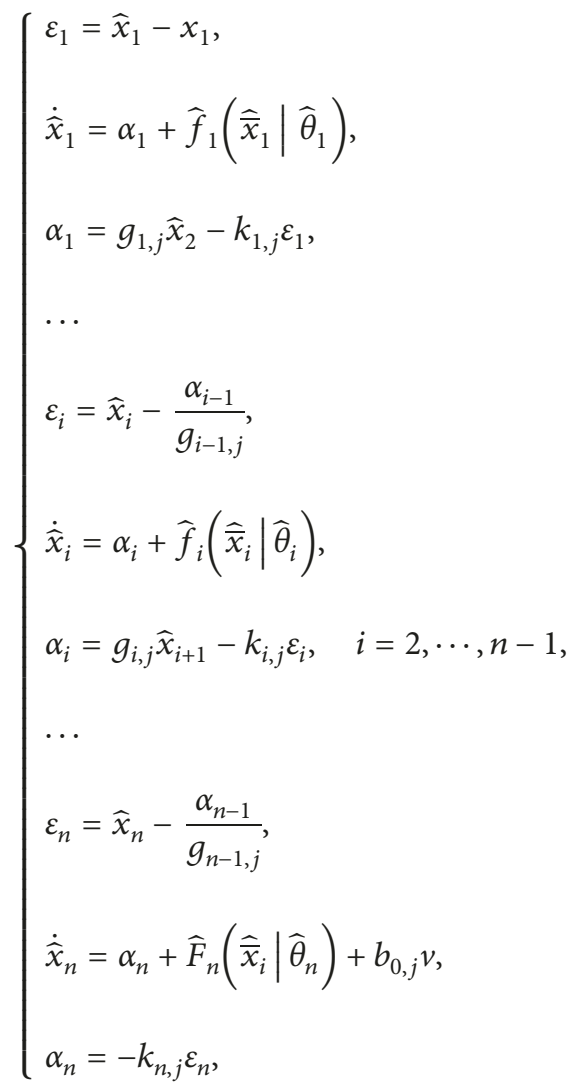

where $\hat{x}=\left[\hat{x}_{1}, \ldots, \hat{x}_{n}\right]^{T}$ is the estimation of real system states. $g_{i, j}=\left[g_{1, j}, \ldots, g_{n, j}\right]^{T}$ and $k_{i, j}=\left[k_{1, j}, \ldots, k_{n, j}\right]^{T}$, $i=1, \ldots, n$, are parameters to be designed. Furthermore, combining (16), the transfer error of the observer $\varepsilon_{i}$ are further expressed as

$$
\left\{\begin{array}{l}
\varepsilon_{1}=\widehat{x}_{1}-x_{1}, \\
\varepsilon_{i}=\frac{\alpha_{i-1} k_{i-1, j}}{g_{i-1, j}} \varepsilon_{i-1}, \\
\varepsilon_{n}=\frac{\alpha_{n-1} k_{n-1, j}}{g_{n-1, j}} \varepsilon_{n-1} .
\end{array}\right.
$$

Further, define the observer error $\bar{e}_{o}$ as

$$
\left\{\begin{array}{l}
e_{o 1}=\widehat{x}_{1}-x_{1} \\
e_{o i}=\hat{x}_{i}-x_{i} \\
e_{o n}=\widehat{x}_{n}-x_{n}
\end{array}\right.
$$

From (16) and (18), the differential with respect to $t$ of $\bar{e}_{o}$ becomes 


$$
\left\{\begin{array}{l}
\dot{e}_{o 1}=g_{1, j} e_{o 2}+\tilde{\theta}_{1}^{T} \phi_{1}\left(\hat{\bar{x}}_{1}\right)+\eta_{1}-k_{1, j} \varepsilon_{1}, \\
\dot{e}_{o i}=g_{i, j} e_{o(i+1)}+\tilde{\theta}_{i}^{T} \phi_{i}\left(\hat{\bar{x}}_{i}\right)+\eta_{i}-k_{i, j} \varepsilon_{i}, \\
\dot{e}_{o n}=\tilde{\theta}_{n}^{T} \phi_{n}\left(\hat{\bar{x}}_{n}\right)+\eta_{n}-k_{n, j} \varepsilon_{n},
\end{array}\right.
$$

where $\widetilde{\theta}_{i}=\theta_{i}-\theta_{i}^{*}(i=1, \ldots, n)$ is the adaptive parameter error.

Define the error transform function $h\left(z_{i}\right)$ as

$$
e_{o i}=\xi h\left(z_{i}\right)
$$

where $h\left(z_{i}\right)=(2 / \pi) \arctan \left(z_{i}\right) \cdot h\left(z_{i}\right)$ is a smooth and strictly increasing function and $h\left(z_{i}\right) \in(-1,1)$. It also satisfies $\lim _{z_{i} \longrightarrow+\infty} h\left(z_{i}\right)=1$ and $\lim _{z_{i} \longrightarrow-\infty} h\left(z_{i}\right)=-1$. Define a new function as

$$
L\left(z_{i}\right)=\frac{1}{2}\left(\frac{d\left(h\left(z_{i}\right)\right)}{d z_{i}} z_{i}+h\left(z_{i}\right)\right),
$$

which satisfies $L\left(z_{a}\right)>L\left(z_{b}\right)$, when $z_{a}>z_{b}$. Combining (20) and (21),

$$
\frac{1}{2}\left(z_{i}+h\left(z_{i}\right)\right)>L\left(z_{i}\right)>\frac{1}{2}\left(h\left(z_{i}\right)\right) .
$$

Differentiating (20) with respect to $t$ yields

$$
\dot{e}_{o i}=\dot{\xi} h\left(z_{i}\right)+\xi \frac{\partial h\left(z_{i}\right)}{\partial z_{i}} \dot{z}_{i}
$$

Then, the dynamic system errors are expressed as

$$
\left\{\begin{aligned}
\dot{z}_{i} & =M\left(\xi, z_{i}\right)\left(g_{i, j} \xi h\left(z_{i+1}\right)-\prod_{i=1}^{n-1} k_{i, j} \xi h\left(z_{1}\right)-\dot{\xi} h\left(z_{i}\right)\right) \\
& +M\left(\xi, z_{i}\right)\left(\widetilde{\theta}_{i}^{T} \phi_{i}\left(\hat{\bar{x}}_{i}\right)+\eta_{i}\right), \\
\dot{z}_{n} & =M\left(\xi, z_{i}\right)\left(-\prod_{i=1}^{n} k_{i, j} \xi h\left(z_{1}\right)-\dot{\xi} h\left(z_{n}\right)\right) \\
& +M\left(\xi, z_{n}\right)\left(\tilde{\theta}_{n}^{T} \phi_{n}\left(\hat{\bar{x}}_{n}\right)+\eta_{n}\right),
\end{aligned}\right.
$$

where $M\left(\xi, z_{i}\right)=1 / \xi\left(\partial h\left(z_{i}\right) / \partial z_{i}\right)$.

Step 1. Construct a Lyapunov function candidate as

$$
V_{i}=\frac{1}{M} z_{i} h\left(z_{i}\right)+\frac{1}{M} z_{i}^{2}, \quad i=1, \ldots, n-1 .
$$

Differentiating (25),

$$
\begin{aligned}
\dot{V}_{i}= & -\left(\frac{1}{M}\right)^{2} \dot{M} z_{i} h\left(z_{i}\right)+\frac{1}{M}\left(\dot{z}_{i} h\left(z_{i}\right)+z_{i} \dot{z}_{i} \dot{h}\left(z_{i}\right)\right) \\
& -\left(\frac{\dot{M}}{M^{2}}\right) z_{i}^{2}+\frac{1}{M} z_{i} \dot{z}_{i} \\
= & -\left(\frac{\dot{M}}{M^{2}}\right) z_{i} h\left(z_{i}\right)-\left(\frac{\dot{M}}{M^{2}}\right) z_{i}^{2} \\
& +\left[g_{i, j} \xi h\left(z_{i+1}\right)-\prod_{i=1}^{i} k_{i} \xi h\left(z_{1}\right)-\dot{\xi} h\left(z_{i}\right)\right] \\
& \cdot\left(h\left(z_{i}\right)+z_{i} \dot{h}\left(z_{i}\right)\right) \\
& +\left(\tilde{\theta}_{i}^{T} \phi_{i}\left(\hat{\bar{x}}_{i}\right)+\eta_{i}\right)\left(h\left(z_{i}\right)+z_{i} \dot{h}\left(z_{i}\right)\right) \\
& +z_{i}\left[g_{i, j} \xi h\left(z_{i+1}\right)-\prod_{i=1}^{i} k_{i, j} \xi h\left(z_{1}\right)-\dot{\xi} h\left(z_{i}\right)\right] \\
& +z_{i}\left(\tilde{\theta}_{i}^{T} \phi_{i}\left(\hat{\bar{x}}_{i}\right)+\eta_{i}\right) .
\end{aligned}
$$

Computing $\quad\left(g_{i, j} \xi h\left(z_{i+1}\right)-\prod_{i=1}^{i} k_{i, j} \xi h\left(z_{1}\right)-\dot{\xi} h\left(z_{i}\right)\right)$ $\left(h\left(z_{i}\right)+z_{i} \dot{h}\left(z_{i}\right)\right)$ gives

$$
\begin{aligned}
\left(g_{i, j} \xi h\left(z_{i+1}\right)-\prod_{i=1}^{i} k_{i, j} \xi h\left(z_{1}\right)-\dot{\xi} h\left(z_{i}\right)\right)\left(h\left(z_{i}\right)+z_{i} \dot{h}\left(z_{i}\right)\right) \\
=2\left[g_{i, j} \xi h\left(z_{i+1}\right)-\prod_{i=1}^{i} \xi h\left(z_{1}\right)-\dot{\xi} h\left(z_{i}\right)\right] L\left(z_{i}\right) \\
\leq g_{i, j} \xi h\left(z_{i+1}\right)\left(z_{i}+h\left(z_{i}\right)\right) \\
\quad-\left(\prod_{i=1}^{i} k_{i, j} \xi h\left(z_{1}\right)+\dot{\xi} h\left(z_{i}\right)\right) h\left(z_{i}\right) .
\end{aligned}
$$

Then, $\left(\tilde{\theta}_{1}^{T} \phi_{1}\left(\hat{\bar{x}}_{1}\right)+\eta_{1}\right)\left(h\left(z_{1}\right)+z_{1} \dot{h}\left(z_{1}\right)\right)$ is rewritten as

$$
\begin{array}{r}
\left(\tilde{\theta}_{i}^{T} \phi_{i}\left(\hat{\bar{x}}_{i}\right)+\eta_{i}\right)\left(h\left(z_{i}\right)+z_{i} \dot{h}\left(z_{i}\right)\right) \\
\leq\left(\widetilde{\theta}_{i}^{T} \phi_{i}\left(\hat{\bar{x}}_{i}\right)+\eta_{i}\right)\left(h\left(z_{i}\right)+z_{i}\right) .
\end{array}
$$
gives

Computing $\quad z_{i}\left[g_{i, j} \xi h\left(z_{i+1}\right)-\prod_{i=1}^{i} k_{i, j} \xi h\left(z_{1}\right)-\dot{\xi} h\left(z_{i}\right)\right]$

$$
\begin{aligned}
& z_{i}\left[g_{i, j} \xi h\left(z_{i+1}\right)-\prod_{i=1}^{i} k_{i, j} \xi h\left(z_{1}\right)-\dot{\xi} h\left(z_{i}\right)\right] \\
& \quad \leq-\left(\prod_{i=1}^{i} k_{i, j} \xi\right) z_{i} h\left(z_{1}\right)-\dot{\xi} z_{i} h\left(z_{i}\right)+g_{i, j} \xi z_{i} h\left(z_{i+1}\right) .
\end{aligned}
$$

Combining (27)-(29) into (26), 


$$
\begin{aligned}
\dot{V}_{i} \leq & -\left(\frac{\dot{M}}{M^{2}}\right) z_{i} h\left(z_{i}\right)-\left(\frac{\dot{M}}{M^{2}}\right) z_{i}^{2} \\
& +g_{i, j} \xi h\left(z_{i+1}\right)\left(z_{i}+h\left(z_{i}\right)\right) \\
& -\left(\prod_{i=1}^{\mathrm{i}} k_{i, j} \xi h\left(z_{1}\right)+\dot{\xi} h\left(z_{i}\right)\right) h\left(z_{i}\right) \\
& -\left(\prod_{i=1}^{\mathrm{i}} k_{i, j} \xi\right) z_{i} h\left(z_{1}\right)-\dot{\xi} z_{i} h\left(z_{i}\right)+g_{i, j} \xi z_{i} h\left(z_{i+1}\right) \\
& +\left(\tilde{\theta}_{i}^{T} \phi_{i}\left(\hat{\bar{x}}_{i}\right)+\eta_{i}\right)\left(h\left(z_{i}\right)+2 z_{i}\right) .
\end{aligned}
$$

In the light of Young's inequality and the characteristic of $h($.$) , we have$

$$
\begin{aligned}
2 h\left(z_{i+1}\right)\left(z_{i}+h\left(z_{i}\right)\right) & \leq 2 h^{2}\left(z_{i+1}\right)+z_{i}^{2}+h^{2}\left(z_{i}\right), \\
2 z_{i} h\left(z_{i+1}\right) & \leq h^{2}\left(z_{i+1}\right)+z_{i}^{2}, \\
h^{2}\left(z_{i}\right) & \leq z_{i}^{2}, \\
\left(\widetilde{\theta}_{i}^{T} \phi_{i}\left(\hat{\bar{x}}_{i}\right)+\eta_{i}\right)\left(h\left(z_{i}\right)+2 z_{i}\right) & \leq\left\|\widetilde{\theta}_{i}\right\|^{2}+\eta_{i}^{2}+3 z_{i}^{2} .
\end{aligned}
$$

By substituting (31) into (30), it follows

$$
\begin{aligned}
\dot{V}_{i} \leq & -\left(\frac{\dot{M}}{M^{2}}+\dot{\xi}\right) z_{i} h\left(z_{i}\right)-\left(\frac{\dot{M}}{M^{2}}+\dot{\xi}-\frac{3 g_{i, j} \xi}{2}-3\right) z_{i}^{2} \\
& +\frac{3 g_{i, j} \xi}{2} z_{i+1}^{2}-\prod_{i=1}^{i} k_{i, j} \xi h\left(z_{1}\right) h\left(z_{i}\right) \\
& -\left(\prod_{i=1}^{i} k_{i, j} \xi\right) z_{i} h\left(z_{1}\right)+\left\|\widetilde{\theta}_{i}\right\|^{2}+\eta_{i}^{2} .
\end{aligned}
$$

Step 2. Consider the Lyapunov function candidate as

$$
V_{n}=\frac{1}{M} z_{n} h\left(z_{n}\right)+\frac{1}{M} z_{n}^{2}
$$

Similar to Step 1,

$$
\begin{aligned}
\dot{V}_{n} \leq & -\left(\frac{\dot{M}}{M^{2}}\right) z_{n} h\left(z_{n}\right)-\left(\frac{\dot{M}}{M^{2}}\right) z_{n}^{2} \\
& -\left(\prod_{i=1}^{n} k_{i, j} \xi h\left(z_{1}\right)+\dot{\xi} h\left(z_{n}\right)\right) h\left(z_{n}\right) \\
& -\left(\prod_{i=1}^{n} k_{i, j} \xi\right) z_{n} h\left(z_{1}\right)-\dot{\xi} z_{n} h\left(z_{n}\right) \\
& +\left(\tilde{\theta}_{n}^{T} \phi_{n}\left(\hat{\bar{x}}_{n}\right)+\eta_{n}\right)\left(h\left(z_{n}\right)+2 z_{n}\right) \\
\leq & -\left(\frac{\dot{M}}{M^{2}}+\dot{\xi}\right) z_{n} h\left(z_{n}\right)-\left(\frac{\dot{M}}{M^{2}}+\dot{\xi}-3\right) z_{n}^{2} \\
& -\prod_{i=1}^{n} k_{i, j} \xi h\left(z_{1}\right) h\left(z_{n}\right)-\left(\prod_{i=1}^{n} k_{i, j} \xi\right) z_{n} h\left(z_{1}\right) \\
& +\left\|\tilde{\theta}_{n}\right\|^{2}+\eta_{n}^{2} .
\end{aligned}
$$

We augment the Lyapunov candidate $V_{o}$ as

$$
V_{o}=\sum_{i=1}^{n} V_{i} \text {. }
$$

Synthesizing (32), (34), and (35),

$$
\dot{V}_{o} \leq-\sum_{i=1}^{n}\left(\frac{\dot{M}}{M^{2}}+\dot{\xi}\right) z_{i} h\left(z_{i}\right)
$$

$$
\begin{aligned}
& -\left(\frac{\dot{M}}{M^{2}}+\dot{\xi}-\frac{3 g_{1, j} \xi}{2}-3\right) z_{1}^{2} \\
& -\sum_{i=2}^{n-1}\left(\frac{\dot{M}}{M^{2}}+\dot{\xi}-\frac{3 g_{i, j} \xi}{2}-\frac{3 g_{i-1, j} \xi}{2}-3\right) z_{i}^{2} \\
& -\left(\frac{\dot{M}}{M^{2}}+\dot{\xi}-\frac{3 g_{n-1, j} \xi}{2}-3\right) z_{n}^{2} \\
& -\sum_{i=1}^{n}\left(\left(\prod_{i=1}^{n} k_{i, j} \xi\right)\left(h\left(z_{1}\right) h\left(z_{i}\right)+z_{i} h\left(z_{1}\right)\right)\right) \\
& +\sum_{i=1}^{n}\left(\left\|\widetilde{\theta}_{n}\right\|^{2}+\eta_{n}^{2}\right) .
\end{aligned}
$$

Stability analysis is described in the following two parts.

Case 1. $z_{1}$ and $z_{i}$ have the same sign (such as both $z_{1}$ and $z_{i}$ are positive or negative). 


$$
\begin{gathered}
\text { Computing }-\left(h\left(z_{1}\right) h\left(z_{i}\right)+z_{i} h\left(z_{1}\right)\right) \text { gives } \\
-\left(h\left(z_{1}\right) h\left(z_{i}\right)+z_{i} h\left(z_{1}\right)\right) \leq 0 .
\end{gathered}
$$

Substituting (37) into (36), one obtains

$$
\begin{aligned}
\dot{V}_{o} \leq & -\sum_{i=1}^{n}\left(\frac{\dot{M}}{M^{2}}+\dot{\xi}\right) z_{i} h\left(z_{i}\right)-\left(\frac{\dot{M}}{M^{2}}+\dot{\xi}-\frac{3 g_{1, j} \xi}{2}-3\right) z_{1}^{2} \\
& -\sum_{i=2}^{n-1}\left(\frac{\dot{M}}{M^{2}}+\dot{\xi}-\frac{3 g_{i, j} \xi}{2}-\frac{3 g_{i-1, j} \xi}{2}-3\right) z_{i}^{2} \\
& -\left(\frac{\dot{M}}{M^{2}}+\dot{\xi}-\frac{3 g_{n-1, j} \xi}{2}-3\right) z_{n}^{2} \\
& -\sum_{i=1}^{n}\left(\left\|\tilde{\theta}_{n}\right\|^{2}+\eta_{n}^{2}\right) .
\end{aligned}
$$

Case 2. $z_{1}$ and $z_{i}$ have opposite signs.

$$
\begin{aligned}
& \text { Computing }-\left(h\left(z_{1}\right) h\left(z_{i}\right)+z_{i} h\left(z_{1}\right)\right) \text { gives } \\
& \begin{aligned}
&-\left(h\left(z_{1}\right) h\left(z_{i}\right)+z_{i} h\left(z_{1}\right)\right) \\
& \quad \frac{1}{2}\left(\left(-h\left(z_{1}\right)\right)^{2}+h^{2}\left(z_{i}\right)+z_{i}^{2}+\left(-h\left(z_{1}\right)\right)^{2}\right) \\
& \quad \leq z_{1}^{2}+z_{i}^{2} .
\end{aligned}
\end{aligned}
$$

Theorem 1. Consider system (5) with unknown backlashlike hysteresis under Definition 1 and error transform function (20), applying the proposed FTSO mechanism in (16), observer errors (18) converge to a small neighborhood of the origin point in a settling time $T_{0}$.

Proof. Substituting (39) into (36), one obtains

$$
\begin{aligned}
\dot{V}_{o} \leq & -\sum_{i=1}^{n}\left(\frac{\dot{M}}{M^{2}}+\dot{\xi}\right) z_{i} h\left(z_{i}\right) \\
& -\left(\frac{\dot{M}}{M^{2}}+\dot{\xi}-\frac{3 g_{1, j} \xi}{2}-3-2 \sum_{i=1}^{n}\left(\prod_{i=1}^{n} k_{i, j} \xi\right)\right) z_{1}^{2} \\
& -\sum_{i=2}^{n-1}\left(\frac{\dot{M}}{M^{2}}+\dot{\xi}-\frac{3 g_{i, j} \xi}{2}-\frac{3 g_{i-1, j} \xi}{2}-3-\sum_{i=1}^{n}\left(\prod_{i=1}^{n} k_{i, j} \xi\right)\right) z_{i}^{2} \\
& -\left(\frac{\dot{M}}{M^{2}}+\dot{\xi}-\frac{3 g_{n-1, j} \xi}{2}-3-\sum_{i=1}^{n}\left(\prod_{i=1}^{n} k_{i, j} \xi\right)\right) z_{n}^{2} \\
& +\sum_{i=1}^{n}\left(\left\|\widetilde{\theta}_{n}\right\|^{2}+\eta_{n}^{2}\right) .
\end{aligned}
$$

For simplification of the expression, reconfigure the parameters $\lambda_{1}=\left(\dot{M} / M^{2}\right)+\dot{\xi}-\left(3 g_{1, j} \xi / 2\right)-3-2 \sum_{i=1}^{n} \prod_{i=1}^{n}$ $k_{i, j} \xi>0, \quad \lambda_{i}=\sum_{i=2}^{n-1}\left(\left(\dot{M} / M^{2}\right)+\dot{\xi}-\left(3 g_{i, j} \xi / 2\right)-\left(3 g_{i-1, j} \xi / 2\right)\right.$ $\left.-3-\sum_{i=1}^{n} \prod_{i=1}^{n} k_{i, j} \xi\right)>0, i=2, \ldots, n$, and $\lambda_{n}=\left(\dot{M} / M^{2}\right)+\dot{\xi}$ $-\left(3 g_{n-1, j} \xi / 2\right)-3-\sum_{i=1}^{n}\left(\prod_{i=1}^{n} k_{i, j} \xi\right)>0$. Futhermore, denote $\lambda=\min \left(\dot{M} / M^{2}\right)+\dot{\xi}, \lambda_{1}, \ldots, \lambda_{n}$ and $D_{o}=\sum_{i=1}^{n}\left(\left\|\widetilde{\theta}_{n}\right\|^{2}+\eta_{n}^{2}\right)$. The Laypunov function $V_{o}$ consequently takes the form

$$
\dot{V}_{o} \leq \lambda V_{o}+D \text {. }
$$

Integrating (41) from 0 to $t$ produces

$$
0 \leq V_{o}(t) \leq \frac{D}{\lambda}+V_{o}(0) e^{-\lambda t} .
$$

Therefore, the observer errors converge to a small neighborhood of the origin point in a finite-time interval $\left[0, T_{0}\right]$, and all of the closed-loop systems signals are bounded.

This completes the proof of Theorem 1.

\section{Adaptive Controller Design and Stability Analysis}

In this section, an adaptive control scheme is developed for nonlinear system with unknown backlash-like hysteresis by using the above FTSO and the backstepping technique. To facilitate the design procedure, the following errors are defined as

$$
\left\{\begin{array}{l}
e_{c 1}=\widehat{x}_{1}-x_{d}, \\
e_{c i}=\widehat{x}_{i}-\gamma_{i-1}, \quad i=2, \cdots, n,
\end{array}\right.
$$

where $\gamma_{i-1}$ are virtual intermediate control signals which will be defined later.

The feedback control laws (44), (45), and adaptive laws (46) are chosen as

$$
\begin{aligned}
& \gamma_{1}=-\frac{e_{c 1} \widehat{\theta}_{1}}{4 \phi_{1}^{T}\left(\overline{\bar{x}}_{1}\right) \phi_{1}\left(\hat{\bar{x}}_{1}\right)}-h_{1, j} e_{c 1}, \\
& \gamma_{i}=-\frac{e_{c i} \widehat{\theta}_{i}}{4 \phi_{i}^{T}\left(\hat{\bar{x}}_{i}\right) \phi_{i}\left(\hat{\bar{x}}_{i}\right)}-h_{i, j} e_{c i}+\frac{\dot{\gamma}_{i-1}}{g_{i, j}}, \quad i=2, \ldots, n, \\
& \dot{\hat{\theta}}_{i}=\frac{d_{i} g_{i, j} e_{c i}^{2}}{4 \phi_{n}^{T}\left(\hat{\bar{x}}_{i}\right) \phi_{i}\left(\hat{\bar{x}}_{i}\right)}-p_{i} \widehat{\theta}_{i}, \quad i=1, \ldots, n,
\end{aligned}
$$

where $v=\gamma_{n} . h_{1, j}>1, d_{1}>0, p_{1}>0, h_{i, j}>1, d_{i}>0$, and $p_{i}>0$ are the design parameters.

Step 1. Consider a Lyapunov candidate function in the form of

$$
V_{c 1}=\frac{1}{2} e_{c 1}^{2}+\frac{1}{2 d_{1}} \tilde{\theta}_{1}^{2},
$$

where $\widetilde{\theta}_{1}=\widehat{\theta}_{1}-\theta_{1}^{*}$, with $\widehat{\theta}_{1}$ being the estimation of $\theta_{1}^{*}=\left\|\hat{\theta}_{1}\right\|^{2}$.

$$
\dot{V}_{c 1}=e_{1} \dot{e}_{1}+\frac{1}{d_{1}} \tilde{\theta}_{1}^{T} \dot{\hat{\theta}}_{1} .
$$

From (16) and (43), one obtains

$$
\begin{aligned}
\dot{e}_{c 1}= & g_{1, j} \widehat{x}_{2}-k_{1, j} \varepsilon_{1}+\hat{f}_{1}\left(\hat{\bar{x}}_{1} \mid \hat{\theta}_{1}\right)-\dot{x}_{d} \\
= & g_{1, j} \hat{x}_{2}-k_{1, j} \varepsilon_{1}+\widehat{\theta}_{1}^{T} \phi_{1}\left(\hat{\bar{x}}_{1}\right) \\
& +\eta_{1}-\dot{x}_{d} \\
= & g_{1, j}\left(e_{c 2}+\gamma_{1}\right)-k_{1, j} \varepsilon_{1}+\widehat{\theta}_{1}^{T} \phi_{1}\left(\hat{\bar{x}}_{1}\right) \\
& +\eta_{1}-\dot{x}_{d} .
\end{aligned}
$$


From (48) and (49), it is obtained that $\dot{V}_{c 1}$ is

$$
\begin{aligned}
\dot{V}_{c 1}= & e_{c 1}\left(g_{1, j}\left(e_{c 2}+\gamma_{1}\right)+\widehat{\theta}_{1}^{T} \phi_{1}\left(\widehat{\bar{x}}_{1}\right)\right) \\
& +e_{c 1}\left(-k_{1, j} \varepsilon_{1}+\eta_{1}-\dot{x}_{d}\right)+\frac{1}{d_{1}} \widetilde{\theta}_{1}^{T} \dot{\hat{\theta}}_{1} .
\end{aligned}
$$

Based on Young's inequality and Lemma 3, the following inequalities are obtained

$$
\begin{aligned}
e_{c 1} \hat{\theta}_{1}^{T} \phi_{1}\left(\hat{\bar{x}}_{1}\right) & \leq \frac{g_{1, j} e_{c 1}^{2}\left\|\theta_{1}\right\|^{2} \phi_{1}^{T}\left(\hat{\bar{x}}_{1}\right) \phi_{1}\left(\hat{\bar{x}}_{1}\right)}{4}+\frac{1}{g_{1, j}} \\
& \leq \frac{g_{1, j} e_{c 1}^{2} \theta_{1}^{*}}{4 \phi_{1}^{T}\left(\hat{\bar{x}}_{1}\right) \phi_{1}\left(\hat{\bar{x}}_{1}\right)}+\frac{1}{g_{1, j}} .
\end{aligned}
$$

Substituting (44), (46), and (51) into (50) gives

$$
\begin{aligned}
\dot{V}_{c 1}= & e_{c 1}\left(g_{1, j}\left(e_{c 2}+\gamma_{1}\right)+\frac{g_{1, j} e_{c 1}\left(\widehat{\theta}_{1}-\widetilde{\theta}_{1}\right)}{4 \phi_{1}^{T}\left(\hat{\bar{x}}_{1}\right) \phi_{1}\left(\hat{\bar{x}}_{1}\right)}\right) \\
& +e_{c 1}\left(-k_{1, j} \varepsilon_{1}+\eta_{1}-\dot{x}_{d}\right)+\frac{1}{d_{1}} \widetilde{\theta}_{1}^{T} \dot{\hat{\theta}}_{1}+\frac{e_{c 1}(0)}{g_{1, j}} \\
& +e_{c 1}\left(g_{1, j}\left(e_{c 2}-h_{1, j} e_{c 1}\right)\right) \\
& +e_{c 1}\left(-k_{1, j} \varepsilon_{1}+\eta_{1}-\dot{x}_{d}\right)+\frac{p_{1}}{d_{1}} \widetilde{\theta}_{1} \widehat{\theta}_{1}+\frac{e_{c 1}(0)}{g_{1, j}} .
\end{aligned}
$$

Via Young's inequality, we have

$$
\begin{aligned}
-\frac{p_{1}}{d_{1}} \widetilde{\theta}_{1} \widehat{\theta}_{1} & \leq-\frac{p_{1}}{2 d_{1}} \widetilde{\theta}_{1}^{2}+\frac{p_{1}}{2 d_{1}} \theta_{1}^{* 2}, \\
e_{c 1}\left(-k_{1, j} \varepsilon_{1}+\eta_{1}-\dot{x}_{d}\right) & \leq \frac{1}{2} e_{c 1}^{2}+\frac{1}{2} \varsigma^{2},
\end{aligned}
$$

where $\varsigma=-k_{1, j} \varepsilon_{1}+\eta_{1}-\dot{x}_{d} \cdot \varepsilon_{1}, \eta_{1}$, and $\dot{x}_{d}$ are bounded.

Thus, (52) is modified as

$$
\dot{V}_{c 1}=-\left(h_{1, j} g_{1, j}-\frac{1}{2}\right) e_{c 1}^{2}-\frac{p_{1}}{2 d_{1}} \tilde{\theta}_{1}^{2}+g_{1, j} e_{c 1} e_{c 2}+E_{1} \text {, }
$$

where $E_{1}=\left(p_{1} / 2 d_{1}\right) \theta_{1}^{* 2}+(1 / 2) \varsigma^{2}+e_{c 1}(0) / g_{1, j}$.

Step $i(i=2, \cdots, n-1)$. Similar to Step 1 , consider a Lyapunov candidate function in the form of

$$
V_{c i}=\frac{1}{2} e_{c i}^{2}+\frac{1}{2 d_{i}} \widetilde{\theta}_{i}^{2}
$$

where $\widetilde{\theta}_{i}=\widehat{\theta}_{i}-\theta_{i}^{*}$.

$$
\dot{V}_{c i}=e_{i} \dot{e}_{i}+\frac{1}{d_{i}} \widetilde{\theta}_{i}^{T} \dot{\hat{\theta}}_{i}
$$

From (43) and (45), one obtains

$$
\dot{e}_{c i}=g_{i, j}\left(e_{c i+1}+\gamma_{i}\right)-\prod_{i=1}^{i} k_{i, j} \varepsilon_{1}+\widehat{\theta}_{i}^{T} \phi_{n}\left(\hat{\bar{x}}_{i}\right)+\eta_{i}-\dot{\gamma}_{i-1} .
$$

From (57) and (58), $\dot{V}_{c i}$ is given by

$$
\begin{aligned}
\dot{V}_{c i}= & e_{c i}\left(g_{i, j}\left(e_{c i+1}+\gamma_{i}\right)+\widehat{\theta}_{i}^{T} \phi_{i}\left(\hat{\bar{x}}_{i}\right)\right) \\
& +e_{c i}\left(-\prod_{i=1}^{i} k_{i, j} \varepsilon_{1}+\eta_{i}-\dot{\gamma}_{i-1}\right)+\frac{1}{d_{i}} \widetilde{\theta}_{i}^{T} \dot{\hat{\theta}}_{i} .
\end{aligned}
$$

Based on Young's inequality and Lemma 3, the following inequalities are obtained:

$$
\begin{aligned}
e_{c i} \hat{\theta}_{i}^{T} \phi_{i}\left(\hat{\bar{x}}_{i}\right) & \leq \frac{g_{i, j} e_{c i}^{2}\left\|\theta_{i}\right\|^{2} \phi_{i}^{T}\left(\hat{\bar{x}}_{i}\right) \phi_{i}\left(\hat{\bar{x}}_{i}\right)}{4}+\frac{1}{g_{i, j}} \\
& \leq \frac{g_{i, j} e_{c i}^{2} \theta_{i}^{*}}{4 \phi_{i}^{T}\left(\hat{\bar{x}}_{i}\right) \phi_{i}\left(\hat{\bar{x}}_{i}\right)}+\frac{1}{g_{i, j}} .
\end{aligned}
$$

Thus,

$$
\begin{aligned}
& \dot{V}_{c i}=e_{c i}\left(g_{i, j}\left(e_{c i+1}-h_{i, j} e_{c i}\right)\right) \\
& +e_{c i}\left(-\prod_{i=1}^{i} k_{i, j} \varepsilon_{1}+\eta_{i}\right)-\frac{p_{i}}{d_{i}} \widehat{\theta}_{i} \widehat{\theta}_{i}+\frac{e_{c i}(0)}{g_{i, j}} .
\end{aligned}
$$

From Young's inequality,

$$
\begin{array}{r}
-\frac{p_{i}}{d_{i}} \widehat{\theta}_{i} \hat{\theta}_{i} \leq-\frac{p_{i}}{2 d_{i}} \tilde{\theta}_{i}^{2}+\frac{p_{i}}{2 d_{i}} \theta_{i}^{* 2}, \\
e_{c i}\left(-\prod_{i=1}^{n-1} k_{i, j} \varepsilon_{1}+\eta_{i}\right) \leq \frac{1}{2} e_{c i}^{2}+\frac{1}{2} \varsigma_{i}^{2},
\end{array}
$$

where $\varsigma_{i}=-\prod_{i=1}^{i} k_{i, j} \varepsilon_{1}+\eta_{i}$ and $\eta_{i}$ are bounded.

Substituting (62) and (63) into (61) gives

$$
\dot{V}_{c i}=-\left(h_{i, j} g_{i, j}-\frac{1}{2}\right) e_{c i}^{2}-\frac{p_{i}}{2 d_{i}} \tilde{\theta}_{i}^{2}+g_{i, j} e_{c i} e_{c i+1}+E_{i}
$$

where $E_{i}=\left(p_{i} / 2 d_{i}\right) \theta_{i}^{* 2}+(1 / 2) \varsigma_{i}^{2}+\left(e_{c i}(0) / g_{i, j}\right)$.

Step $n$. Similar to Step $i$, consider a Lyapunov candidate function in the form of

$$
V_{c n}=\frac{1}{2} e_{c n}^{2}+\frac{1}{2 d_{n}} \widetilde{\theta}_{n}^{2}
$$

where $\tilde{\theta}_{n}=\widehat{\theta}_{n}-\theta_{n}^{*}$.

Similar to Step $i$, one obtains

$$
\dot{e}_{c n}=-\prod_{i=1}^{n} k_{i, j} \varepsilon_{1}+\widehat{\theta}_{n}^{T} \phi_{n}\left(\widehat{\bar{x}}_{n}\right)+\eta_{n}-\dot{\gamma}_{n-1}+b_{0, j} \nu \text {. }
$$

Moreover, the time-derivative of $V_{c n}$ is given by

$$
\begin{aligned}
\dot{V}_{c n}= & e_{c n}\left(b_{0, j} v+\widehat{\theta}_{n}^{T} \phi_{n}\left(\hat{\bar{x}}_{n}\right)\right) \\
& +e_{c n}\left(-\prod_{i=1}^{n} k_{i, j} \varepsilon_{1}+\eta_{n}-\dot{\gamma}_{n-1}\right)+\frac{1}{d_{n}} \tilde{\theta}_{n}^{T} \dot{\hat{\theta}}_{n} .
\end{aligned}
$$

Based on Young's inequality and Lemma 3, the following inequalities are obtained: 


$$
\begin{aligned}
e_{c n} \hat{\theta}_{n}^{T} \phi_{n}\left(\hat{\bar{x}}_{n}\right) & \leq \frac{b_{0, j} e_{c n}^{2}\left\|\theta_{n}\right\|^{2} \phi_{n}^{T}\left(\hat{\bar{x}}_{n}\right) \phi_{n}\left(\hat{\bar{x}}_{n}\right)}{4}+\frac{1}{b_{0, j}} \\
& \leq \frac{b_{0, j} e_{c n}^{2} \theta_{n}^{*}}{4 \phi_{n}^{T}\left(\overline{\bar{x}}_{n}\right) \phi_{n}\left(\overline{\bar{x}}_{n}\right)}+\frac{1}{b_{0, j}} .
\end{aligned}
$$

Substituting (45), (67), and (68) gives

$$
\begin{aligned}
\dot{V}_{c n}= & e_{c n}\left(b_{0, j}\left(-h_{n, j} e_{c n}\right)\right)+e_{c n}\left(-\prod_{\mathrm{i}=1}^{\mathrm{n}} k_{n, j} \varepsilon_{1}+\eta_{n}\right) \\
& -\frac{p_{n} \widetilde{\theta}_{n} \widehat{\theta}_{n}+\frac{e_{c n}(0)}{b_{0, j}}}{}
\end{aligned}
$$

By using Young's inequality,

$$
\begin{array}{r}
-\frac{p_{n}}{d_{n}} \widetilde{\theta}_{n} \widehat{\theta}_{n} \leq-\frac{p_{n}}{2 d_{n}} \widetilde{\theta}_{n}^{2}+\frac{p_{n}}{2 d_{n}} \theta_{n}^{* 2}, \\
e_{c n}\left(-\prod_{\mathrm{i}=1}^{\mathrm{n}} k_{i, j} \varepsilon_{1}+\eta_{n}\right) \leq \frac{1}{2} e_{c n}^{2}+\frac{1}{2} \varsigma_{n}^{2},
\end{array}
$$

where $\varsigma_{n}=-\prod_{i=1}^{n} k_{i, j} \varepsilon_{1}+\eta_{n}$ and $\eta_{n}$ are bounded.

Thus, (69) is modified to be

$$
\dot{V}_{c n}=-\left(h_{n, j} b_{0, j}-\frac{1}{2}\right) e_{c n}^{2}-\frac{p_{n}}{2 d_{n}} \tilde{\theta}_{n}^{2}+E_{n},
$$

where $E_{n}=\left(p_{n} / 2 d_{n}\right) \theta_{n}^{* 2}+(1 / 2) \varsigma_{n}^{2}+\left(e_{c n}(0) / b_{0, j}\right)$.

A Lyapunov function $V_{c}$ is chosen as

$$
V_{c}=\sum_{i=1}^{n} V_{c i}=\sum_{i=1}^{n} \frac{1}{2} e_{c i}^{2}+\sum_{i=1}^{n} \frac{1}{2 d_{i}} \widetilde{\theta}_{i}^{2} .
$$

The differentiation of equation (73) is

$$
\begin{aligned}
\dot{V}_{c}= & \sum_{i=1}^{n} \dot{V}_{c i} \\
\leq & -\sum_{i=1}^{n-1}\left(h_{i, j} g_{i, j}-\frac{1}{2}\right) e_{c i}^{2}-\sum_{i=1}^{n} \frac{p_{i}}{2 d_{i}} \tilde{\theta}_{i}^{2} \\
& +\sum_{i=1}^{n-1} g_{i, j} e_{c i} e_{c i+1}+\sum_{i=1}^{n} E_{i} \\
& -\left(h_{n, j} b_{0, j}-\frac{1}{2}\right) e_{c n}^{2} .
\end{aligned}
$$

Based on Young's inequality, it is obtained that

$$
\begin{aligned}
g_{i, j} e_{c i} e_{c i+1} & \leq g_{i, j}\left(\frac{h_{i, j}}{2} e_{c i}^{2}+\frac{1}{2 h_{i}} e_{c i+1}^{2}\right) \\
& \leq g_{i, j}\left(\frac{h_{i, j}}{2} e_{c i}^{2}+\frac{1}{2} e_{c i+1}^{2}\right),
\end{aligned}
$$

with $h_{i, j}>1$.
Thus, equation (74) is modified as

$$
\begin{aligned}
\dot{V}_{c} \leq & -\sum_{i=1}^{n-1}\left(h_{i, j} g_{i, j}-\frac{1}{2}\right) e_{c i}^{2}-\sum_{i=1}^{n} \frac{p_{i}}{2 d_{i}} \tilde{\theta}_{i}^{2} \\
& +\sum_{i=1}^{n-1} g_{i, j}\left(\frac{h_{i, j}}{2} e_{c i}^{2}+\frac{1}{2} e_{c i+1}^{2}\right) \\
& +\sum_{i=1}^{n} E_{i}-\left(h_{n, j} b_{0, j}-\frac{1}{2}\right) e_{c n}^{2} \\
\leq & -\frac{1}{2} \sum_{i=1}^{n-1}\left(h_{n, j} g_{i, j}-2\right) e_{c i}^{2}-\frac{1}{2}\left(2 h_{n, j} b_{0, j}-2\right) e_{c n}^{2} \\
& -\sum_{i=1}^{n} \frac{p_{i}}{2 d_{i}} \widetilde{\theta}_{i}^{2}+\sum_{i=1}^{n} E_{i} .
\end{aligned}
$$

Furthermore, denote $\mu=\min \left\{h_{1, j} g_{1, j}-2, \cdots, h_{n-1, j} g_{n-1, j}-2\right.$, $\left.2 h_{n, j} b_{0, j}-2, p_{1}, p_{2}, \cdots, p_{n}\right\}$ and $E_{c}=\sum_{i=1}^{n} E_{i}$. The Laypunov function $V_{c}$ consequently takes the form of

$$
\dot{V}_{c} \leq-\mu V_{c}+E_{c} \text {. }
$$

Theorem 2. For switched strict-feedback nonlinear systems with unknown hysteresis (1), FTSO (16), feedback control laws (44) and (45), and adaptive laws (46), if the average dwell time of the switching signal $\sigma(t)$ satisfies the condition $\tau_{a}>$ $(\ln \zeta / \mu)\left(\zeta=\max \left\{\lambda_{\max }\left(P_{k}\right) / \lambda_{\min }\left(P_{l}\right)\right\}, k, l \in M\right)$, the control scheme based on FTSO ensures that all variables of the closedloop system are bounded. By properly selecting the parameters, the controller and observer error converge to origin, such that $\lim _{T \rightarrow \infty} z_{1}^{2} \leq 0$ and $\lim _{T \rightarrow \infty} e_{1}^{2} \leq 0$.

Proof. There is a piecewise differentiable function $W(t)=$ $e^{\mu t} V_{j}(\varepsilon(t))$ in any interval $\left[t_{i-1}, t_{i}\right]$. Then, its time differentiation on $\left[t_{i-1}, t_{i}\right]$ is

$$
\dot{W}(t)=\mu e^{\mu t} V_{j}(\varepsilon(t))+e^{\mu t} \dot{V}_{j}(\varepsilon(t)) .
$$

From (76) and (78),

$$
\dot{W}(t) \leq E_{c} e^{\mu t} .
$$

Then, noting that at the switching point $V\left(t_{i}\right) \leq \zeta V\left(t_{i}^{*}\right)$ from Lemma 2, where $t_{i}^{*}=\lim _{t \rightarrow t_{i}} t$, it follows that

$$
\begin{aligned}
W\left(t_{i}\right) & =e^{\mu t_{i}} V_{\sigma\left(t_{i}\right)}\left(\varepsilon\left(t_{i}\right)\right) \\
& \leq \zeta e^{\mu t_{i}} V_{\sigma\left(t_{i-1}\right)}+\left(\varepsilon\left(t_{i}\right)\right)=\zeta W\left(t_{i}^{*}\right) \\
& \leq \zeta\left(W\left(t_{i-1}\right)+\int_{t_{i-1}}^{t_{i}} E_{c} e^{\mu t} \mathrm{~d} t\right) .
\end{aligned}
$$

Let $T_{f}>t_{0}=0$ and $i \in\left[1, N\left(0, T_{f}\right)\right]$, then equation (80) is further expressed as 


$$
\begin{aligned}
W\left(T_{f}^{*}\right) \leq & W\left(t_{N\left(0, T_{f}\right)}\right)+\int_{t N\left(0, T_{f}\right)}^{T_{f}} E_{c} e^{\mu t} \mathrm{~d} t \\
\leq & \zeta\left[W\left(t_{N\left(0, T_{f}\right)^{-1}}\right)+\int_{t N}^{t N\left(0, T_{f}\right)} E_{c} e^{\mu t} \mathrm{~d} t\right] \\
& +\int_{t N\left(0, T_{f}\right)}^{T_{f}} E_{c} e^{\mu t} \mathrm{~d} t \\
\leq & \zeta^{2}\left[W\left(t_{N\left(0, T_{f}\right)^{-2}}\right)+\int_{t N}^{t N\left(0, T_{f}\right)-2} E_{c} e^{\mu t} \mathrm{~d} t\right] \\
& +\zeta \int_{t N}^{t N\left(0, T_{f}\right)} E_{c} e^{\mu t} \mathrm{~d} t \\
& +\int_{t N}^{T_{f}\left(0, T_{f}\right)} E_{c} e^{\mu t} \mathrm{~d} t \\
\leq & \cdots \\
\leq & \zeta^{N\left(0, T_{f}\right)_{W(0)}} \\
& +\zeta^{N\left(0, T_{f}\right)} \sum_{i=1}^{N\left(0, T_{f}\right)+1} \zeta^{1-i} \int_{t_{i-1}}^{t_{i}} E_{c} e^{\mu t} \mathrm{~d} t .
\end{aligned}
$$

Since $\tau_{a}>(\ln \zeta / \mu)$ and Lemma 1 , for any $T_{f}>0$,

$$
N\left(0, T_{f}\right) \leq N_{0}+\frac{\mu T_{f}}{\ln \zeta}
$$

Substituting (82) from (81),

$$
\begin{aligned}
W\left(T_{f}^{*}\right) \leq & \zeta^{N\left(0, T_{f}\right)} W(0)+\zeta^{N\left(0, T_{f}\right)} \int_{0}^{T_{f}} E_{c} e^{\mu t} \mathrm{~d} t \\
\leq & \zeta^{N_{0}} e^{-\mu T_{f}} W(0) \\
& +\zeta^{N_{0}} e^{-\mu T_{f}} \frac{E_{c}}{\mu}\left(e^{\mu T_{f}}-1\right) .
\end{aligned}
$$

By Lemma 2 and (83), one has

$$
\begin{aligned}
\chi_{1}(\|\varepsilon(T)\|) \leq & V_{\sigma\left(T^{*}\right)}\left(\varepsilon\left(T^{*}\right)\right) \\
= & \zeta^{N_{0}} e^{-\mu T} W(0)+\zeta^{N_{0}} \frac{E_{c}}{\mu} \frac{\left(1-e^{-\mu T}\right)}{e^{\mu t}} \\
\leq & e^{N_{0} \ln \zeta} e^{-2 \mu T} \chi_{2}(\|\varepsilon(0)\|) \\
& +\zeta^{N_{0}} e^{-\mu T} \frac{E_{c}}{\mu}\left(1-e^{-\mu T}\right) .
\end{aligned}
$$

Therefore, from (84), it follows that

$$
\lim _{T \longrightarrow \infty} z_{1}^{2} \leq e^{N_{0} \ln \zeta} e^{-2 \mu T} \chi_{2}(\|\varepsilon(0)\|)+\zeta^{N_{0}} e^{-\mu T} \frac{\kappa}{\mu}\left(1-e^{-\mu T}\right)=0 .
$$

Similarly, one obtains

$$
\lim _{T \longrightarrow \infty} e_{1}^{2} \leq 0
$$

That is, using (85) and (86), observation errors and tracking errors converge to zero.

\section{Simulation Results}

In this section, three examples are considered to test the proposed control method. Consider the following secondorder nonlinear system:

$$
\left\{\begin{array}{l}
\dot{x}_{1}=g_{1, \sigma(t)} x_{2}+f_{1, \sigma(t)}\left(\bar{x}_{1}\right)+d_{1, \sigma(t)}(t), \\
\dot{x}_{2}=g_{2, \sigma(t)} u+f_{2, \sigma(t)}\left(\bar{x}_{2}\right)+d_{2, \sigma(t)}(t),
\end{array}\right.
$$

where $g_{1,1}=1, \quad g_{1,2}=1, \quad f_{1,1}\left(\bar{x}_{1}\right)=\left(x_{1}-x_{1}^{3}\right) /\left(1+x_{1}^{4}\right)$, $f_{1,1}\left(\bar{x}_{1}\right)=\left(x_{1}-x_{1}^{2}\right) /\left(1+x_{1}^{5}\right), g_{2,1}=1, g_{2,2}=1, f_{2,1}\left(\bar{x}_{2}\right)=$ $-\exp \left(-x_{1}^{2}\right) \sin \left(5 x_{2}\right), \quad f_{2,1}\left(\bar{x}_{2}\right)=-\sin \left(5 x_{2}\right), \quad u(t)=v(t)+$ $l(v), \quad l(v)=\left(u_{0}-v_{0}\right) \exp \left(-5\left(v-v_{0}\right) \operatorname{sgn} \dot{v}\right)+\exp (-5 v \operatorname{sgn} \dot{v})$ $\int_{v_{0}}^{v}(0.345-1) \exp \left(5 \xi_{\operatorname{sgn} v}\right) d \xi, \quad d_{1,1}(t)=0.2 \sin t, \quad d_{1,2}(t)=$ $0.3 \sin t, d_{2,1}(t)=0.2 \cos t$, and $d_{2,2}(t)=0.3 \cos t$.

The selection guidelines of the design parameters in the proposed control design method are summarized in the following:

Step 1. During selection of design parameters of observer (16), the parameter $k_{i, j}>0$ are selected before $k_{i+1, j}>0$. Then, the values are usually chosen to ensure $k_{i, j}<k_{i+1, j}$.

Step 2. Determine the parameters of finite-time performance function (15) in FTSO (16). In this step, one needs to choose finite-time $T_{0}=0.3$, the initial values $\xi(0)=0.99$, and the final value $\xi_{T 0}=0.01$. Then, $\tau=$ 0.5 and $\lambda=6.66$ are chosen. Moreover, it is obtained that $T_{0}=\xi(0) / \tau \lambda$.

Step 3. Select controller parameters of $h_{i, j}>1, d_{i}>0$, and $p_{i}>0$ to ensure $\mu>0$, which is defined in (76).

Step 4. Initial values of the system states and observer are set according to the proof of Theorem 1. The appropriate initial conditions are given in order to make the contrast effect of control and observation more clear. In this simulation, $x(0)=[00]^{T}$ and $\widehat{x}(0)=\left[\begin{array}{ll}0 & 0\end{array}\right]^{T}$.

For better illustration, we consider such two cases: (1) the proposed adaptive controller scheme with FTSO and (2) the proposed adaptive controller scheme with the observer in [12]. The reference signals $x_{d}$ are selected for $x_{d}=2$ and $x_{d}=1.5 \sin 2 t$. The basis function vector $\phi(x)$ is chosen as the form of the Gaussian function:

$$
\Phi_{i}(\bar{x})=\exp \left[-\frac{\left(\bar{x}-\mu_{i}\right)^{T}\left(\bar{x}-\mu_{i}\right)}{2 \gamma_{i}^{2}}\right],
$$

with five nodes, the width $\gamma_{1}=0.5$ and $\gamma_{2}=0.8$, and the centers $\mu_{i}(i=1,2)$ are equally distributed between $[2,2]$.

The chosen control parameters are $d_{1}=1, d_{2}=1, p_{1}=2$, $p_{2}=2, h_{1,1}=30, h_{1,2}=30, h_{2,1}=3$, and $h_{2,2}=9$ and $x(0)=$ $\left[\begin{array}{ll}0 & 0\end{array}\right]^{T}, \widehat{x}(0)=\left[\begin{array}{ll}0 & 0\end{array}\right]^{T}$, and $\widehat{\theta}(0)=\left[\begin{array}{ll}0 & 0\end{array}\right]^{T}$. The FTSO parameters are selected as $k_{1,1}=5.1, k_{1,2}=5.2, k_{2,1}=9$, and 


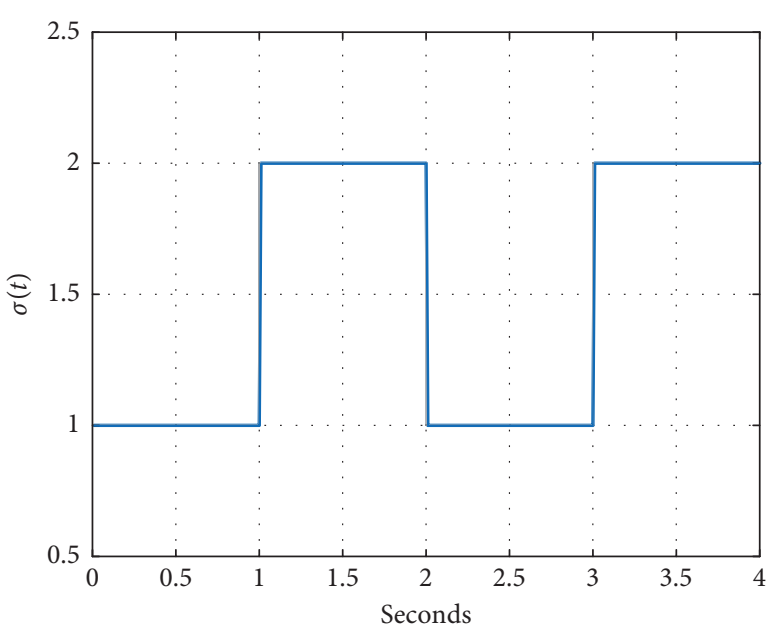

FIgURE 2: Switching signal $\sigma(t)$ in simulation.

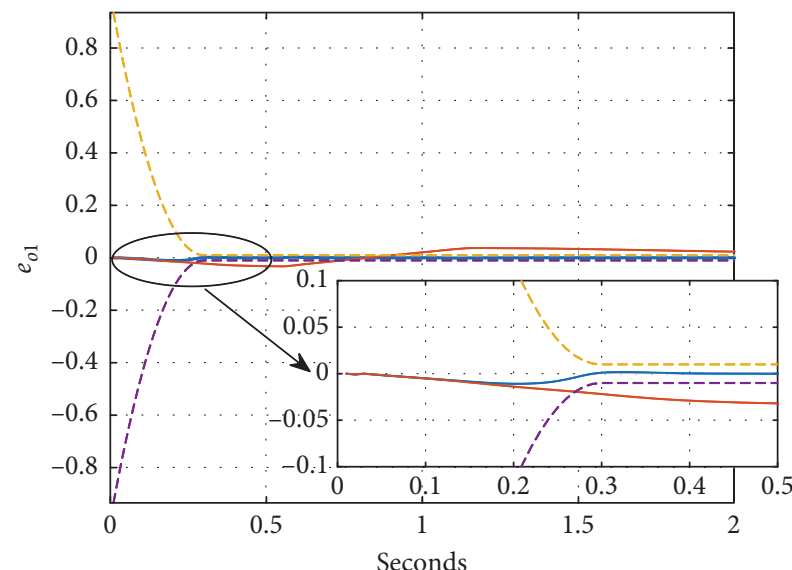

$\begin{array}{ll}\text { _ The FTSO in this work } & \text { - - } \text { Upper bound } \\ \text { _ The observer in [12] } & \text { - - } \text { Lower bound }\end{array}$

Figure 3: The observer compared results of $e_{o 1}$.

$k_{2,2}=9.5$, and the other observer parameters are $k_{1,1}=10$, $k_{1,2}=11, k_{2,1}=21$, and $k_{2,2}=20$.

Example 1. $x_{d}=2$.

Switching signal $\sigma(t)$ is shown in Figure 2, and the observer errors $e_{o 1}$ with the FTSO and the observer in [12] are shown in Figure 3. The tracking performance of the switched system are shown in Figure 4, while the tracking errors $e_{c 1}$ and the control input $v$ of two schemes are shown in Figures 5 and 6, respectively.

\section{Example 2. $x_{d}=1.5 \sin 2 t$.}

The tracking responses and errors with the FTSO and the contrastive observer are depicted in Figure 7. Furthermore, in order to further analyze the performance of the tracking effect with the same controller in Figure 7, the performance evaluation, including average tracking error and average fluctuation of tracking error, is summarized in Table 1.

Figures 8 and 9 show the observer error $e_{o 1}$ compared and the control input $v$ for two cases, respectively.

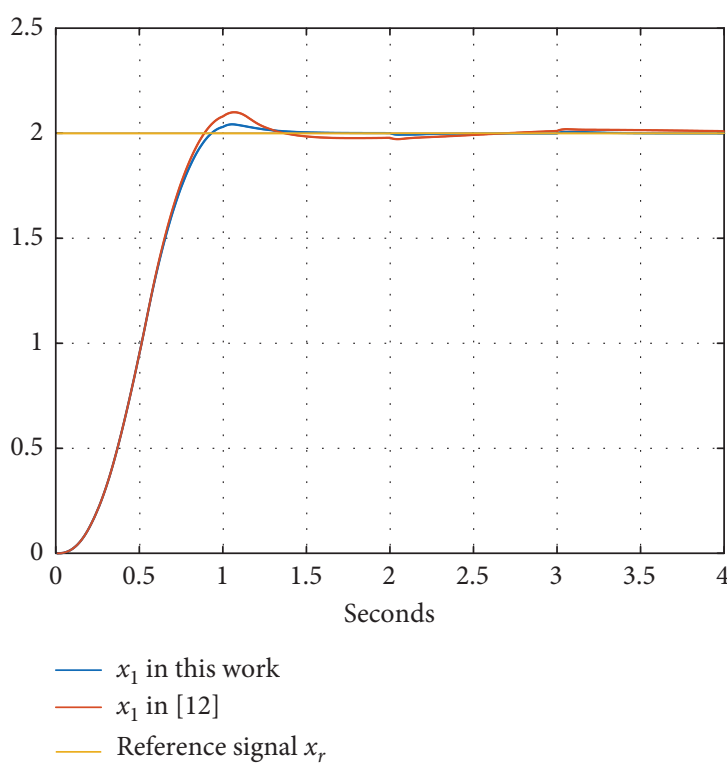

FIgURE 4: The observer compared results of $x_{1}$.

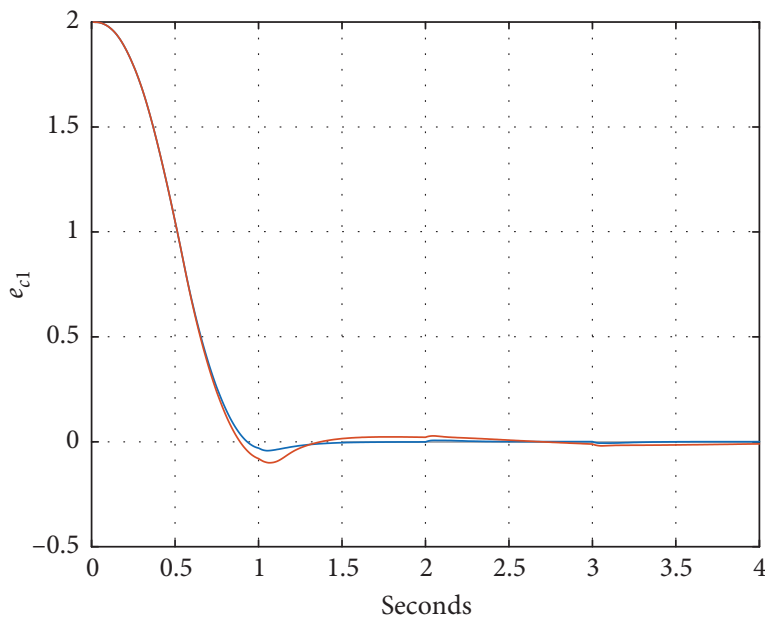

- Tracking error $e_{c 1}$ in this work Tracking error $e_{c 1}$ in [12]

FIgURE 5: The observer compared results of $e_{c 1}$.

Example 3. In order to provide the convictive proof of the proposed control approach, a test is proceeded on a servo test rig (as shown in Figure 10), which is given as follows:

$$
\left\{\begin{array}{l}
J_{m} \ddot{x}_{1}=-b_{m} \dot{x}_{1}-\tau_{s}+\tau_{m} \\
J_{l} \ddot{x}_{2}=-b_{l} \dot{x}_{2}+\tau_{s}-\tau_{d}
\end{array}\right.
$$

where $x_{1}$ and $x_{2}$ denote the displacement of motor subsystem and the load subsystem, respectively. $\tau_{s}$ is the transmitted torque. $\tau_{m}$ is the driving torque of the system, and $\tau_{d}$ is the disturbance torque at the driven gear. $J_{m}=0.01, J_{l}=0.05, b_{m}=0.1$, and $b_{l}=0.12$.

The reference signal $y_{d}=0.8 \sin (t)(\mathrm{rad})$, where parameters are selected as $h_{1}=4, h_{2}=6, k_{1}=200$, and $k_{2}=200$. The initial state of the switched system and observer are $x_{1}(0)=0, x_{2}(0)=0, \widehat{x}_{1}(0)=0$, and $\widehat{x}_{2}(0)=0$. 


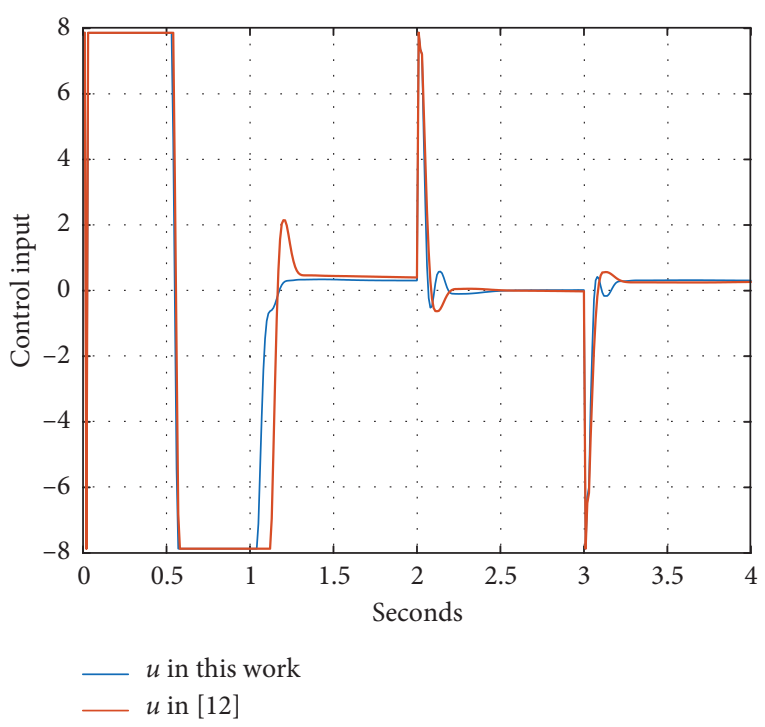

Figure 6: Control input $v$.

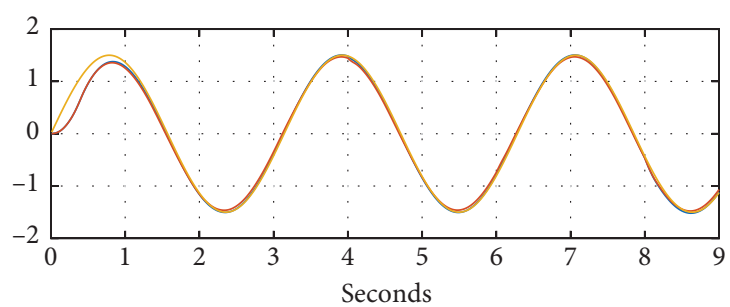

- $x_{1}$ in this work

- $x_{1}$ in [12]

_ Reference signal $x_{r}$

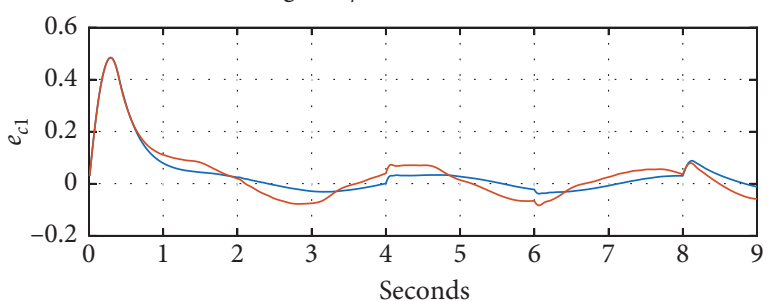

- Tracking error $e_{c 1}$ in this work

_ Tracking error $e_{c 1}$ in [12]

FIGURE 7: The output tracking responses of $x_{1}$ and the tracking error $e_{c 1}$.

TABle 1: Position tracking performance evaluation.

\begin{tabular}{lcc}
\hline Method & $\begin{array}{c}\text { Average } \\
\text { tracking error }\end{array}$ & $\begin{array}{c}\text { Fluctuation of } \\
\text { tracking error }\end{array}$ \\
\hline With the FTSO & 0.0362 & 0.5220 \\
With the observer in [12] & 0.0571 & 1.6707 \\
\hline
\end{tabular}

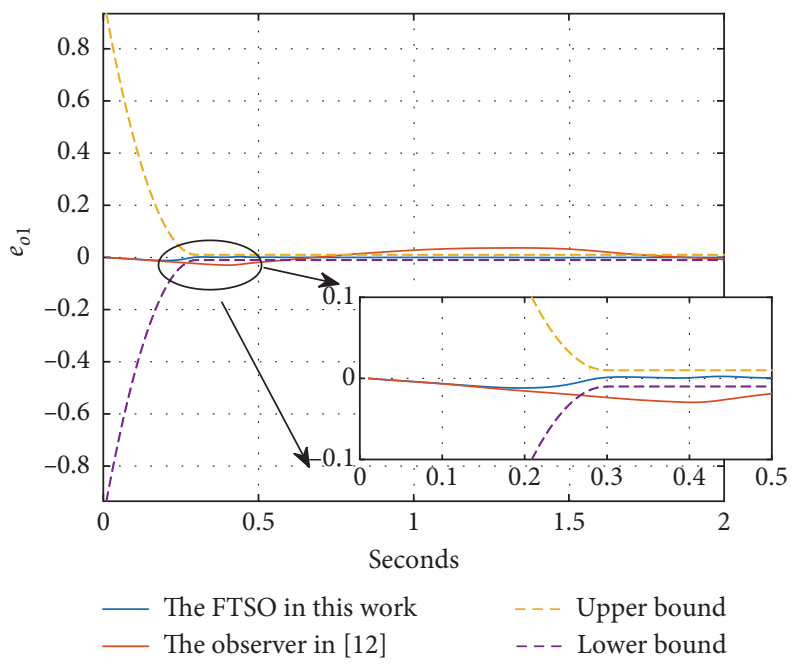

FIgURE 8: The observer compared results of $e_{o 1}$.

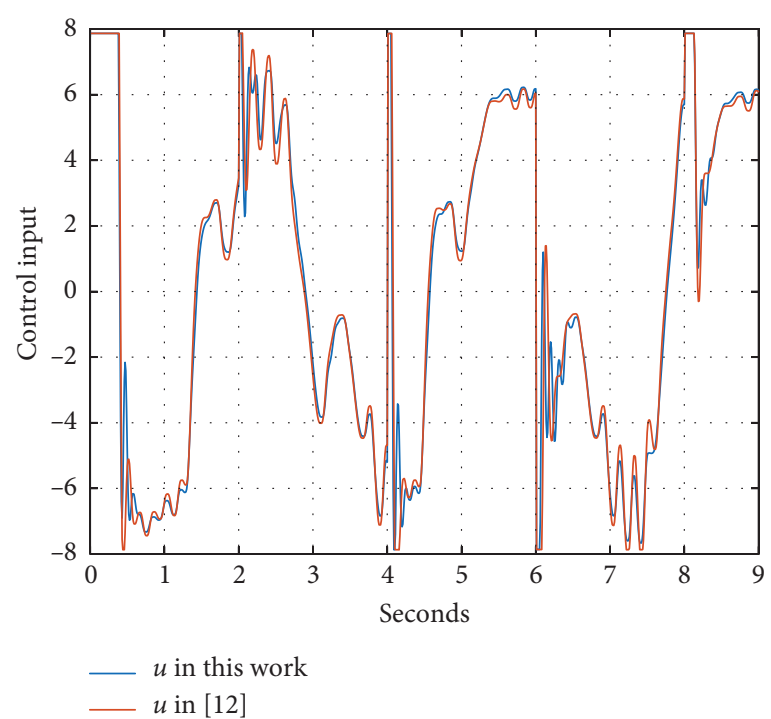

Figure 9: Control input $v$.

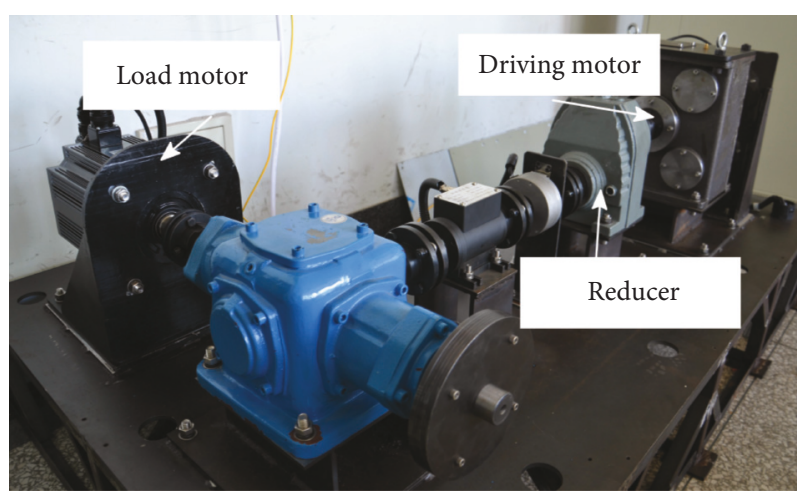

FIgURE 10: Motor driving test rig. 

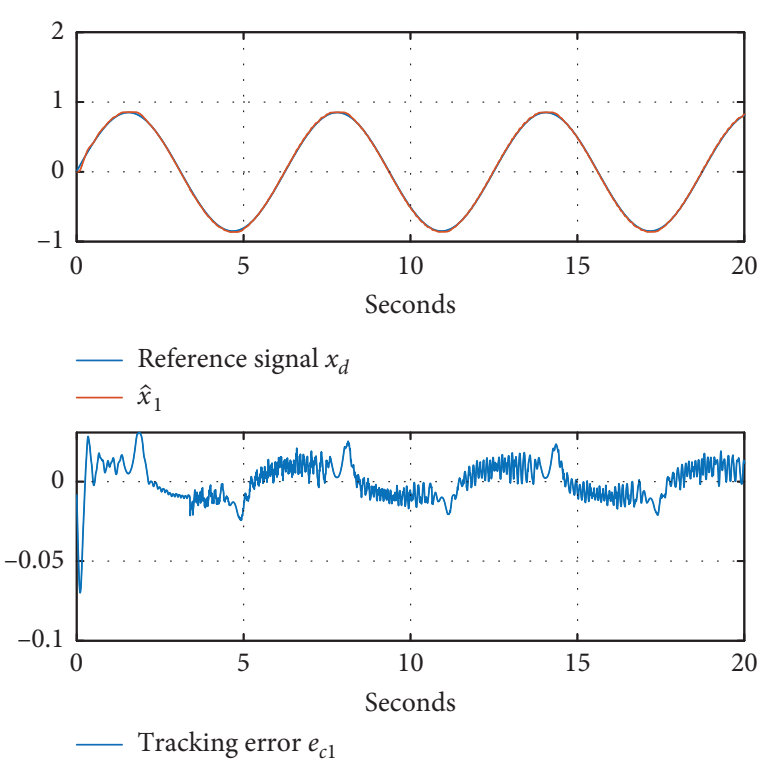

Figure 11: Tracking effect and tracking error.

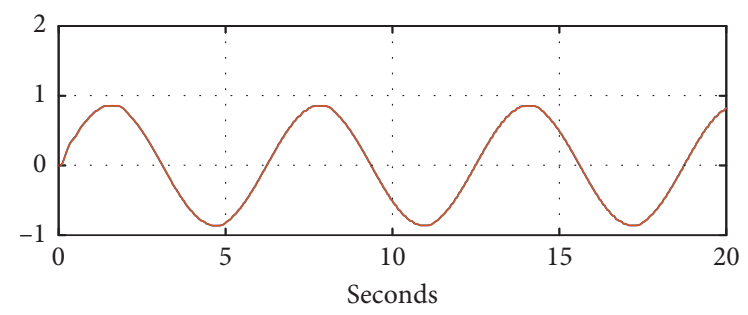

$-\hat{x}_{1}$

- System output $x_{1}$

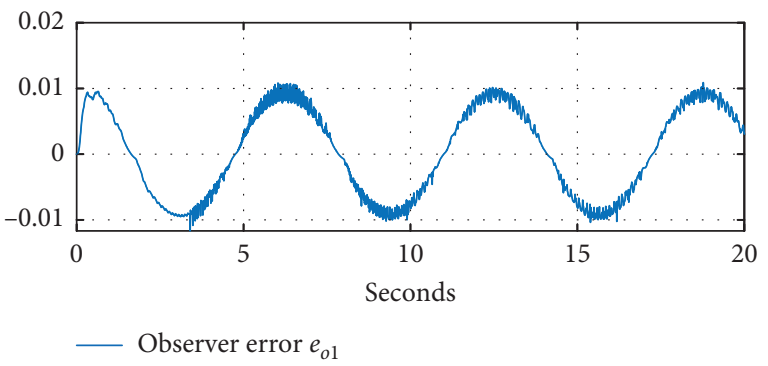

FIGURE 12: Observer effect and error.

The trajectory shown in Figure 11 is the estimation of output $\hat{x}_{1}$ and reference signal $x_{d}$ and the tracking error. Figure 12 shows the trajectories of system output $x_{1}$ and $\hat{x}_{1}$ and the error between system output and estimation of output.

\section{Conclusion}

In this paper, the adaptive switched control with a finitetime switched state observer has been investigated for a class of uncertain nonlinear systems with unknown backlash-like hysteresis in a strict-feedback form. A finitetime switched state observer has been adopted to estimate the unmeasurable states and obtain a better tracking performance, with the help of finite-time performance function and neural network technologies. By transforming the tracking error into a virtual variable and based on the backstepping technique, an adaptive control method has been proposed. It has been proved that the observer error is bounded and finite time is convergent with the proposed control scheme. Moreover, the signals of the resulting system are bounded and the output tracking error is convergent to a small compact set of the origin.

\section{Data Availability}

The data used to support the findings of this study are available from the corresponding author upon request.

\section{Conflicts of Interest}

The authors declare that they have no conflicts of interest.

\section{Acknowledgments}

This work was supported by the National Natural Science Foundation of China under Grant nos. 61703224, 61620304, and 21606141.

\section{References}

[1] Q. Chen, X. Ren, J. Na, and D. Zheng, “Adaptive robust finitetime neural control of uncertain PMSM servo system with nonlinear dead zone," Neural Computing and Applications, vol. 28, no. 12, pp. 3725-3736, 2017.

[2] W. Lv and F. Wang, "Finite-time adaptive fuzzy tracking control for a class of nonlinear systems with unknown hysteresis," International Journal of Fuzzy Systems, vol. 20, no. 3, pp. 782-790, 2018.

[3] S. Li, X. Peng, Y. Tang, and Y. Shi, "Finite-time synchronization of time-delayed neural networks with unknown parameters via adaptive control," Neurocomputing, vol. 308, no. 25 , pp. 65-74, 2018.

[4] J. Xia, J. Zhang, W. Sun, B. Zhang, and Z. Wang, "Finite-time adaptive fuzzy control for nonlinear systems with full state constraints," IEEE Transactions on Systems, Man, and Cybernetics: Systems, vol. 49, no. 7, pp. 1541-1548, 2019.

[5] F. Wang, B. Chen, C. Lin, J. Zhang, and X. Meng, "Adaptive neural network finite-time output feedback control of quantized nonlinear systems," IEEE Transactions on Cybernetics, vol. 48, no. 6, pp. 1839-1848, 2018.

[6] Y. Li, K. Li, and S. Tong, "Finite-time adaptive fuzzy output feedback dynamic surface control for MIMO nonstrict feedback systems," IEEE Transactions on Fuzzy Systems, vol. 27, no. 1, pp. 96-110, 2019.

[7] Z. Yu, Y. Yang, S. Li, and J. Sun, "Observer-based adaptive finite-time quantized tracking control of nonstrict-feedback nonlinear systems with asymmetric actuator saturation," IEEE Transactions on Systems, Man, and Cybernetics: Systems, pp. 1-12, 2018.

[8] Y. Liu, X. Liu, Y. Jing, and Z. Zhang, "A novel finite-time adaptive fuzzy tracking control scheme for nonstrict feedback systems," IEEE Transactions on Fuzzy Systems, vol. 27, no. 4, pp. 646-658, 2019.

[9] S. Sui, C. L. P. Chen, and S. Tong, "Neural network filtering control design for nontriangular structure switched nonlinear 
systems in finite time," IEEE Transactions on Neural Networks and Learning Systems, vol. 30, no. 7, pp. 2153-2162, 2019.

[10] F. Wang, B. Chen, Y. Sun, and C. Lin, "Finite time control of switched stochastic nonlinear systems," Fuzzy Sets and Systems, vol. 365, no. 15, pp. 140-152, 2019.

[11] Q. Chen, M. Gao, L. Tao, and Y. Nan, "Adaptive fixed time parameter estimation and synchronization control for multiple robotic manipulators," International Journal of Control, Automation and Systems, vol. 17, no. 9, pp. 2375-2387, 2019.

[12] M.-M. Jiang and X.-J. Xie, "Adaptive finite-time stabilisation of high-order uncertain nonlinear systems," International Journal of Control, vol. 91, no. 10, pp. 2159-2169, 2018.

[13] Q. Chen, X. Ren, and J. Na, "Robust finite-time chaos synchronization of uncertain permanent magnet synchronous motors," ISA Transactions, vol. 58, pp. 262-269, 2015.

[14] Q. Chen, S. Xie, M. Sun, and X. He, "Adaptive nonsingular fixed-time attitude stabilization of uncertain spacecraft," IEEE Transactions on Aerospace and Electronic Systems, vol. 54, no. 6, pp. 2937-2950, 2018.

[15] M. Tao, Q. Chen, X. He, and M. Sun, “Adaptive fixed-time fault-tolerant control for rigid spacecraft using a double power reaching law," International Journal of Robust and Nonlinear Control, vol. 29, no. 12, pp. 4022-4040, 2019.

[16] V. Hassani, T. Tjahjowidodo, and T. N. Do, "A survey on hysteresis modeling, identification and control," Mechanical Systems and Signal Processing, vol. 49, no. 1-2, pp. 209-233, 2014.

[17] Z. Li, J. Shan, and U. Gabbert, "Inverse compensation of hysteresis using Krasnoselskii-Pokrovskii model," IEEE/ASME Transactions on Mechatronics, vol. 23, no. 2, pp. 966-971, 2018.

[18] I. Mayergoyz, "Mathematical models of hysteresis," IEEE Transactions on Magnetics, vol. 22, no. 5, pp. 603-608, 1986.

[19] X. Zhang, Z. Li, C.-Y. Su, and Y. Lin, "Robust adaptive outputfeedback control for a class of nonlinear systems with hysteresis compensation controller," International Journal of Adaptive Control and Signal Processing, vol. 31, no. 11, pp. 1636-1654, 2017.

[20] W. Lv, F. Wang, and Y. Li, "Finite-time adaptive fuzzy outputfeedback control of MIMO nonlinear systems with hysteresis," Neurocomputing, vol. 296, pp. 74-81, 2018.

[21] Y. Jian, D. Huang, J. Liu, and D. Min, "High-precision tracking of piezoelectric actuator using iterative learning control and direct inverse compensation of hysteresis," IEEE Transactions on Industrial Electronics, vol. 66, no. 1, pp. 368377, 2019.

[22] L. Ma, X. Huo, X. Zhao, B. Niu, and G. Zong, "Adaptive neural control for switched nonlinear systems with unknown backlash-like hysteresis and output dead-zone," Neurocomputing, vol. 357, no. 10, pp. 203-214, 2019.

[23] C. Li and X. Yang, "Adaptive neural output feedback control for a class of switched non-linear systems with unknown backlash-like hysteresis of the actuator," Transactions of the Institute of Measurement and Control, vol. 41, no. 4, pp. 900-910, 2019.

[24] X.-Y. Zhang and Y. Lin, "A robust adaptive dynamic surface control for nonlinear systems with hysteresis input," Acta Automatica Sinica, vol. 36, no. 9, pp. 1264-1271, 2010.

[25] S. Sui, S. Tong, C. L. P. Chen, and K. Sun, "Fuzzy adaptive optimal control for nonlinear switched systems with actuator hysteresis," International Journal of Adaptive Control and Signal Processing, vol. 33, no. 4, pp. 609-625, 2019.

[26] Y. Wang, H. Lei, J. Ye, X. Bu, and Y. Xue, "Guaranteeing prescribed performance control for gyrostabilized platform with unknown control direction preceded by hysteresis,"
International Journal of Aerospace Engineering, vol. 2019, pp. 1-12, 2019.

[27] J. Na, Q. Chen, X. Ren, and Y. Guo, “Adaptive prescribed performance motion control of servo mechanisms with friction compensation," IEEE Transactions on Industrial Electronics, vol. 61, no. 1, pp. 486-494, 2014.

[28] M. Wang, X. Ren, Q. Chen, S. Wang, and X. Gao, "Modified dynamic surface approach with bias torque for multi-motor servomechanism," Control Engineering Practice, vol. 50, pp. 57-68, 2016.

[29] M. Wang, X. Ren, and Q. Chen, "Robust tracking and distributed synchronization control of a multi-motor servomechanism with H-infinity performance," ISA Transactions, vol. 72, no. 1, pp. 147-160, 2018.

[30] C. Wei, M. Benosman, and T. Kim, "Online parameter identification for state of power prediction of lithium-ion batteries in electric vehicles using extremum seeking," International Journal of Control, Automation and Systems, pp. 1-11, 2019.

[31] D. Chowdhury, Y. Al-Nadawi, and X. Tan, "Hysteresis compensation using extended high-gain observer and dynamic inversion," ASME Proceedings \& Mechatronics, no. DSCC2018-9082, 9 pages, 2018.

[32] X. Huo, L. Ma, X. Zhao, B. Niu, and G. Zong, "Observer-based adaptive fuzzy tracking control of MIMO switched nonlinear systems preceded by unknown backlash-like hysteresis," Information Sciences, vol. 490, pp. 369-386, 2019.

[33] J. Wang, K. Chen, Q. Liu, and Q. Ma, "Observer-based adaptive consensus tracking control for nonlinear multi-agent systems with actuator hysteresis," Nonlinear Dynamics, vol. 95, no. 3, pp. 2181-2195, 2019.

[34] X. Wang, X. Yin, and F. Shen, "Disturbance observer based adaptive neural prescribed performance control for a class of uncertain nonlinear systems with unknown backlash-like hysteresis," Neurocomputing, vol. 299, pp. 10-19, 2018.

[35] Y. Feng, C. A. Rabbath, T. Chai, and C. Su, "Robust adaptive control of systems with hysteretic nonlinearities: a Duhem hysteresis modelling approach," in Proceedings of the 2009 IEEE Conference on Africon, Nairobi, Kenya, September 2010.

[36] G. Zhai, B. Hu, K. Yasuda, and A. Michel, "Piecewise Lyapunov functions for switched systems with average dwell time," Asian Journal of Control, vol. 2, no. 3, pp. 192-197, 2000.

[37] G. Lai, Z. Liu, Y. Zhang, C. L. P. Chen, and S. Xie, “Adaptive inversion-based fuzzy compensation control of uncertain pure-feedback systems with asymmetric actuator backlash," IEEE Transactions on Fuzzy Systems, vol. 25, no. 1, pp. 141$155,2017$.

[38] S. Tong, Y. Li, and S. Sui, "Adaptive fuzzy tracking control design for SISO uncertain nonstrict feedback nonlinear systems," IEEE Transactions on Fuzzy Systems, vol. 24, no. 6, pp. 1441-1454, 2016.

[39] Q. Chen, L. Shi, J. Na, X. Ren, and Y. Nan, “Adaptive echo state network control for a class of pure-feedback systems with input and output constraints," Neurocomputing, vol. 275, no. 1, pp. 1370-1382, 2018. 


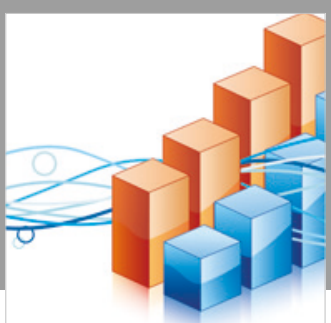

Advances in

Operations Research

\section{-n-m}
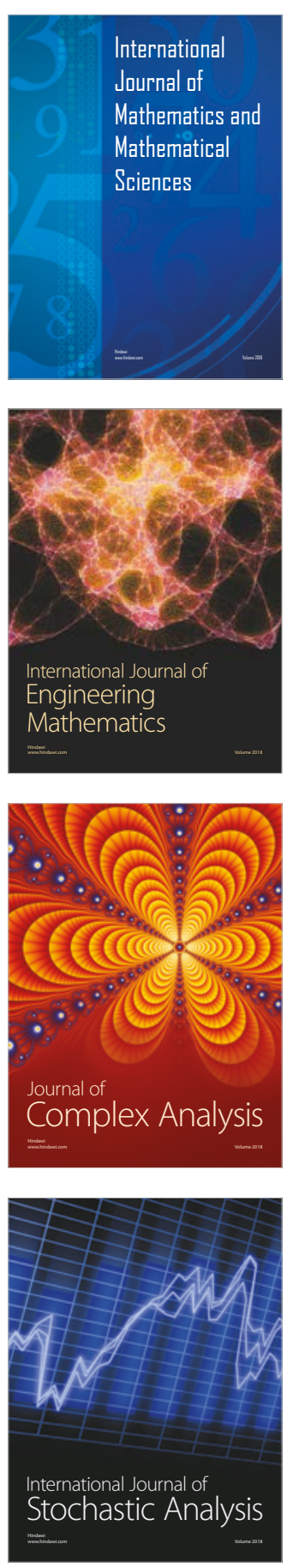
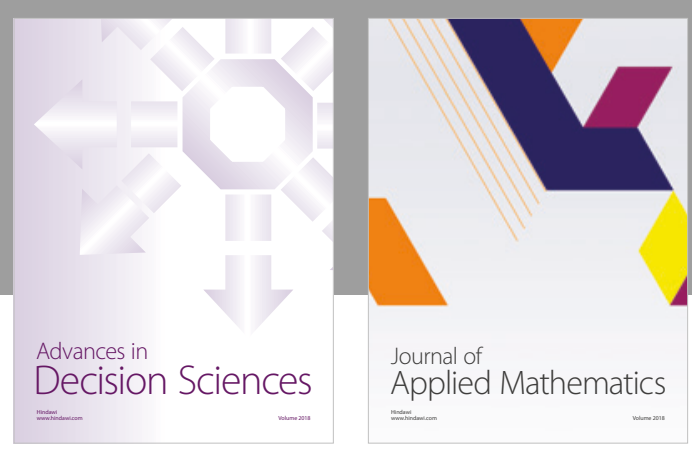

Journal of

Applied Mathematics
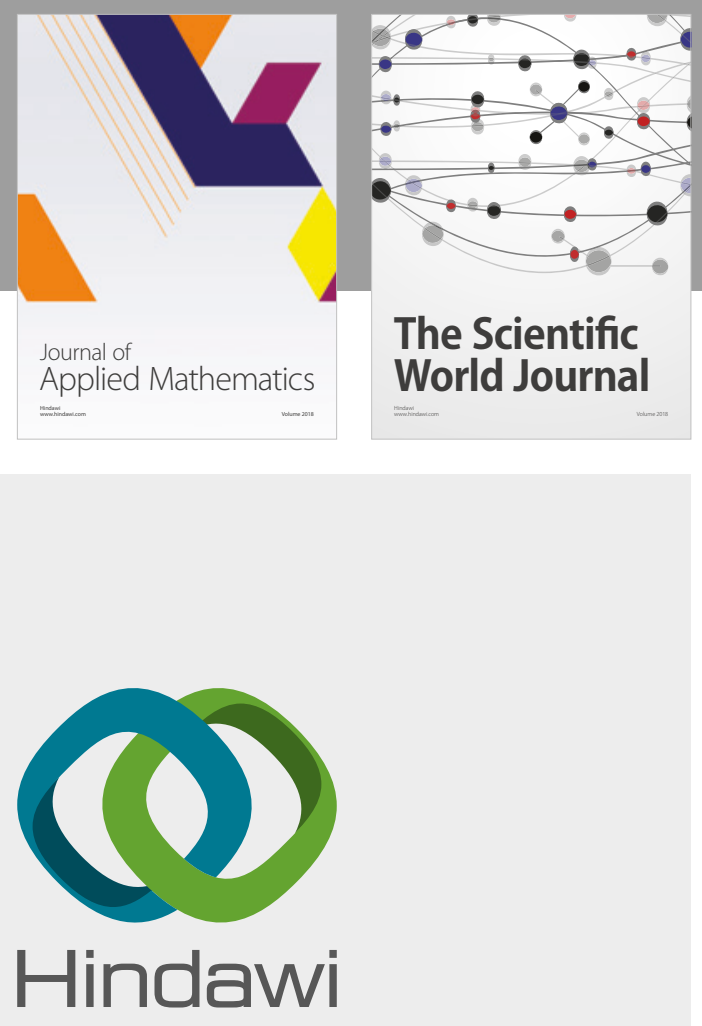

Submit your manuscripts at

www.hindawi.com

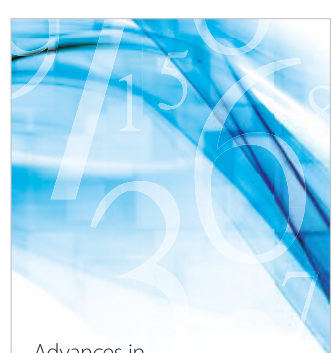

Advances in
Numerical Analysis
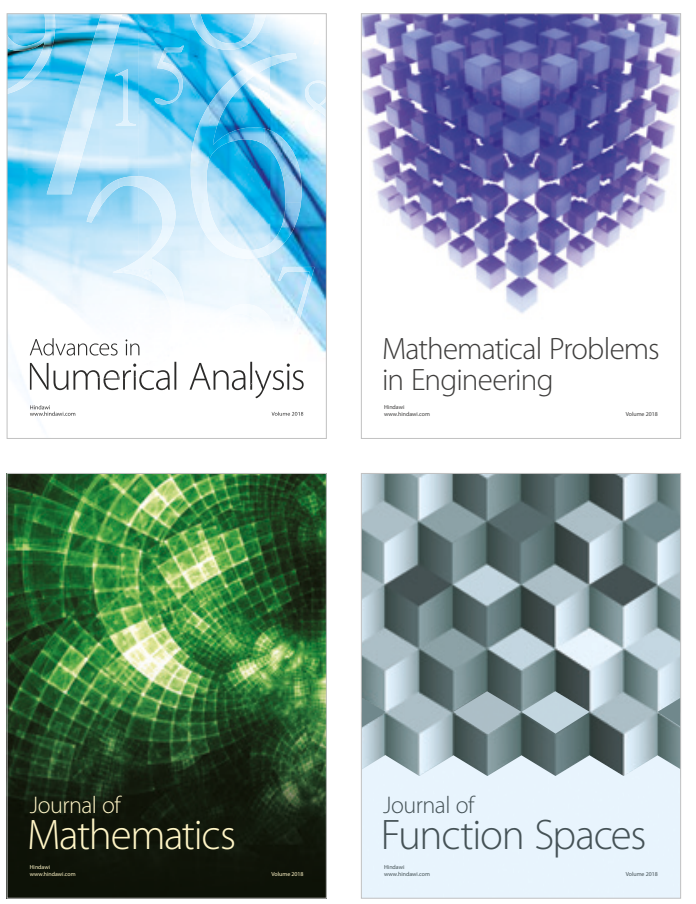

Mathematical Problems in Engineering

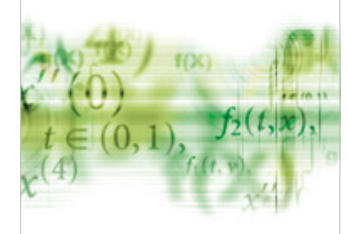

International Journal of

Differential Equations

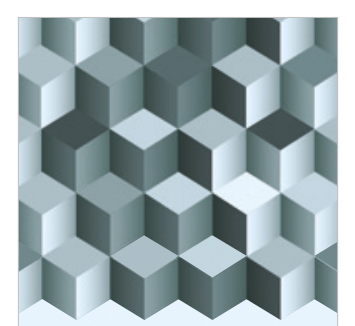

Journal of

Function Spaces
The Scientific

World Journal

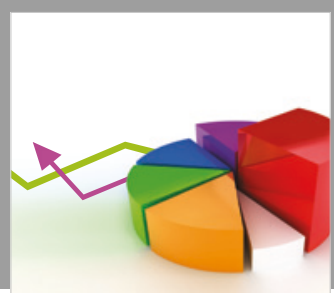

Journal of

Probability and Statistics
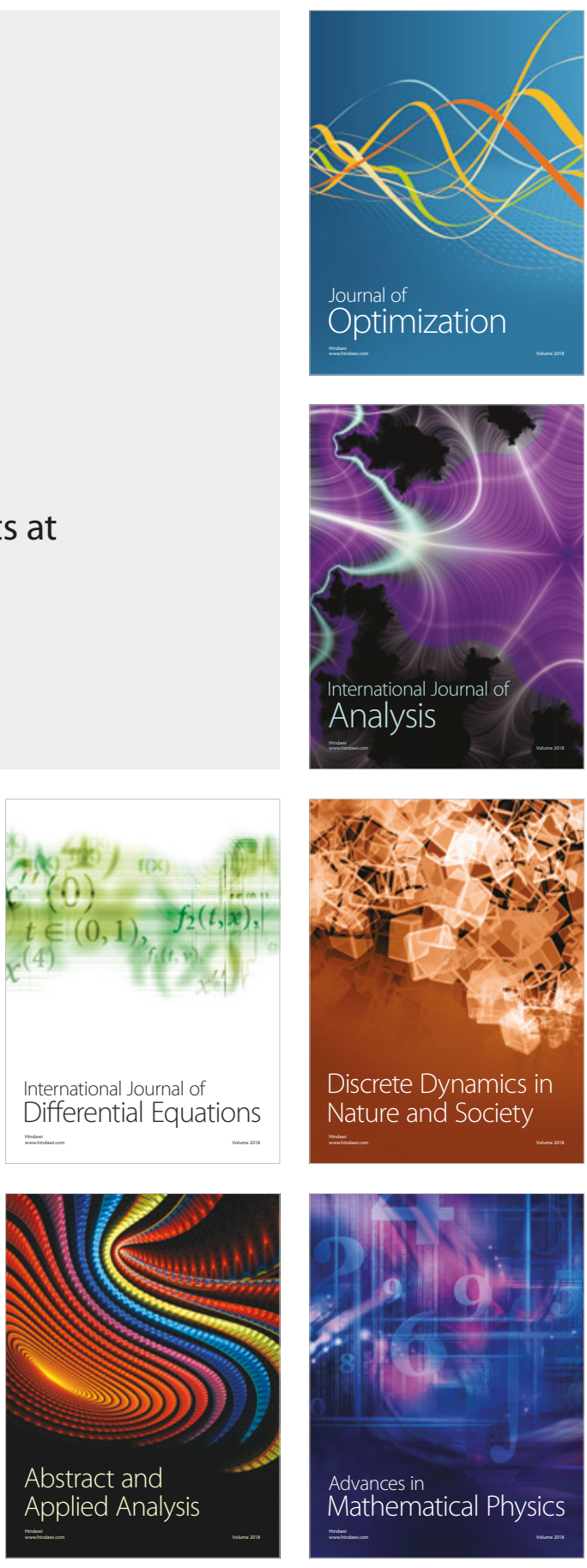